Original Research Paper

\title{
Climate Change-An Unprecedented Challenge for Humankind Survival; Energy Exploitation from the Atmospheric Electrical Circuit
}

\author{
${ }^{1,2}$ Giovanni Pietro Gregori and ${ }^{2}$ Bruce Allen Leybourne \\ ${ }^{1}$ IDASC-Istituto di Acustica e Sensoristica O. M. Corbino (CNR), Roma, Now Merged into IMM-Istituto \\ per la Microelettronica e Microsistemi (CNR) and ISSO-International Seismic Safety Organization, Italy \\ ${ }^{2}$ GeoPlasma Research Institute-(GeoPlasmaResearchInstitute.org), Aurora, CO 80014, Italy
}

Article history

Received: 12-03-2021

Revised: 28-03-2021

Accepted: 04-04-2021

Corresponding Author: Giovanni P. Gregori IDASC-Istituto di Acustica e Sensoristica O. M. Corbino (CNR), Roma, Now Merged into IMM-Istituto per la Microelettronica e Microsistemi (CNR) and ISSOInternational Seismic Safety Organization, Italy

Email: gregori.giovanni@fastwebnet.it
Abstract: A more detailed summary is given in the last part (1/4th) of the Introduction. An anomalous period is in progress, characterized by a very intense release of endogenous energy from the Earth. The cause is an ongoing heartbeat of the Earth's electrocardiogram. Humankind's history spans a much shorter duration. Hence, at present the humans are going to face an unprecedented challenge, maybe even concerning survival. The duration of the climate anomaly of an Earth's heartbeat is a few Ma. The challenge is to avoid that humankind is going toward extinction. The paper attempts to give a general-and largely original and substantially advanced-perspective of Earth's phenomena. As far as possible, the approach is top-down, i.e., start from known laws and infer expected observations-unlike the more usual bottom-up viewpoint, i.e., start from observations and seek regularities. The present text is devoted to applied scientists. The overall scenario is much more focused and precise than standard treatments. The target of the paper is concerned with the possible actions that can-and must-be implemented in order to face this unprecedented challenge-rather than being concerned with a critical discussion and comparison with the present "generally agreed" geophysical models reported in the literature. In particular, the exploitation is considered of a huge reservoir of natural and clean energy. The central focus of the present paper is the exploitation of the electrostatic energy of the atmospheric electrical circuit, which is a clean and almost inexhaustible free source. It should be stressed that-as it has been proven by quantitative and precise observations-the $\mathrm{CO}_{2}$ pollution of anthropic origin provides an irrelevant-or maybe even a negligible-contribution to climate change. Climate change is, rather, caused by the natural, violent, ongoing exhalation of endogenous energy. This anomaly is going to be manifested, perhaps, by an eventual ever-increasing intensity of phenomena-including seismic and volcanic activity, energy and dynamics on the oceans and atmosphere, greater excursions with respect to the mild climate and a (presently unmonitored) change of endemic microorganism generation on the ocean floors (maybe even affecting pandemics). This study is an attempt to synthesize a wide multidisciplinary scenario, as far as possible by a limited number of pages. The purpose is only to start a much more extensive and profound discussion, that-as far as the geophysical items are concerned-is the object of more extensive studies (in preparation).

Keywords: Climate Change, Clean Energy Exploitation, Optimum Locations, Atmospheric Electrical Circuit, Mid-Ocean Ridges, Soil Exhalation, Stellar Transformer, Earth's Electrocardiogram, Endogenous Energy, Geodynamo, Earth's Battery 


\section{Introduction}

\section{A Matter of Anomalous Soil Exhalation}

Great achievements of applied science rely on quantum mechanics, independent of the unsettled conceptual controversies between Schrödinger's and Bohr's schools and others. Even more striking, the Sagnac effect, which seems to be forgotten in treatises on relativity, was exploited by applied science and the optical-fiber gyroscope was thus implemented (MacKenzie, 1993).

The present paper aims to provide applied scientists and technologists with an unconventional account-as brief as possible-of some recent developments and evidences in Earth's sciences. An applied Earth scientist can look at various facets, including the exploitation of a huge natural reservoir of "clean" and free energy, i.e., the electrostatic energy of the atmosphere.

But, a key relevant concern is about the hazard of humankind, while facing an unprecedented challenge.

An event is in progress and it must be managed, asmaybe-it could even imply the extinction of several species and the birth of new species. Therefore, the challenge might even threaten the survival of humankind. It is likely that unprecedented actions are to be considered or eventually required or recommended.

The focus of the present paper is on such a perspective.

Owing to brevity purposes, the related extensive critical discussion is carried out by other treatments (either available ${ }^{1}$ or in preparation).

Just two premises: One semantic and one methodological.

The term "climate" is here defined as the "spacetime domain where the biosphere can develop and survive". That is, "climate" is not just statistics of environmental data. Statistics and "science" are both useful, but they cannot be identified with each other, much like the WHO data must be distinguished from medical science. That is, statistics of phenomena can give a hunch. But, "science" is concerned with explanation of processes and mechanisms, in order to manage possible hazards.

Concerning methods, in general the better-known approach to Earth's science is bottom-up, i.e., start from observations and try and guess an explanation. The approach here adopted is, rather, mainly top-down, i.e., start from known laws and infer the observations to be expected. Bottom-up is exploratory analysis, top-down is confirmatory analysis and only the combination of the two is "science" (Tukey, 1977).

It must be stressed, however, that-consistently with the Ockham razor-the present top-down model wants to avoid, as much as possible, every additional speculation. On the other hand, one must consider that several

${ }^{1}$ Owing to the several topics that are here briefly mentioned, it is impossible to give any extensive list of references. Only a few indications are given. The reader will kindly understand.

2 "Battery" is every device that stores energy. It can rely or a capacitor, or on reversible chemical reactions. The Earth's battery phenomena can be explained also by some alternative more or less intricate mechanisms that, a priori, ought never to be excluded. However, every additional hypothesis should be checked through confirmatory analysis, to be exploited by means of observations. If no such a confirmation is available, one cannot include a mere speculation into an essentially self-consistent, reasonably exhaustive and simple top-down interpretation. A simple top-down interpretation is the first basic target.

The key challenge is the ongoing violent climate change. The cause is certainly natural. The Earth regularly displays an "electrocardiogram", with a pace of $27.4 \mathrm{Ma}$. Every heartbeat lasts some 4-5 Ma and is characterized by a huge amount of endogenous heat release. Hence, humankind-that since prehistory spans, say, $\sim 30.000$ years-is experiencing for the first time such a phenomenon. This is a true threat and a challenge.

The timescale must always be taken into account. The socalled "terminations", that are recorded in ice-cores, are recurrent features lasting several $10^{4}$ years, with a typical "saw-tooth" trend, but are just minor additional details.

That is, the often-reported concern about the climate change provided by ice cores, spanning, say, $\sim 400,000$ years, is an almost irrelevant detail compared to the 27.4 Ma pace of the electrocardiogram.

All major extinctions always occurred during the most paroxysmal stages of heartbeats. For instance, one major case history occurred two heartbeats ago, i.e., some $\sim 55$ Ma ago, or $\sim 2$ hbu ago, where hbu denotes a "heartbeat unit" (27.4 Ma), which is the natural pace that marks Earth's history. At that time, during a few million years, the Arctic region was covered by subtropical vegetation-and the present $\mathrm{CH}_{4}$, oil and coal mining are a signature of that event.

The electrocardiogram can be explained as follows. The natural mechanism of climate change relies on a violent increase in soil exhalation. The Earth operates like a battery ${ }^{2}$ that during almost $\sim 27.4 \mathrm{Ma}$ is recharged. In the meantime, a "channel" is progressively opened, through which energy can be released. As soon as the "channel" is fully open, a huge release of energy occurs and the battery discharges. Hence, the deep Earth cools and the "channel" is closed, while the battery starts to recharge and a new $\sim 27.4$ Ma cycle begins. The pace is an internal process, while the external modulation of the process is considered in the section on A Leading Endogenous Energy Source.

The anomalous release of endogenous energy implies a huge amount of geothermal and fluid exhalation, with an impact on climate and on the biosphere (endemic generation of unprecedented microorganisms), while geodynamics and seismicity are strongly enhanced, in addition to volcanism. Note, however, that volcanoes

relies, rather, on a change of the state-of-matter, that is observed as a change vs. time of the radius of the Inner Core Boundary (ICB) See the section on A Leading Endogenous Energy Source. 
respond with some irregular time-delay, as every volcano operates like the security valve of a pressure cooker, where the valve is represented by the volcanic edifice.

Note that the present-often reported by mass mediafashionable concern about anthropic $\mathrm{CO}_{2}$ is an independent concern and, in any case, it has been proven to be a false dread. Therefore, it is certainly wise to limitand to refrain from every action that is the source of-some unneeded environmental pollution of any kind (unless the human action is aimed to improve climate). But it is certainly a false hope that humankind can face the challenge of climate change by focusing on a kind of pollution that is certainly irrelevant-or maybe even negligible. Correct priorities must be tackled.

The present paper is organized as follows:

The first item is focused on a leading endogenous energy source: That is, the discussion deals with the origin of the endogenous energy, the battery behavior of the Earth, the Earth's electrocardiogram, the energy balance of the Earth, the effects on climate and on geodynamics, seismicity, volcanism, etc., including the modulation by the solar wind, that is perturbed by the encounters of the Solar System with intergalactic clouds, inside a perspective of Galaxy-Sun-Earth Relations.

Then, the mechanism is discussed of heat transport from the deep source through Earth's surface.

The control of climate is the next topic, as it is achieved by three natural engines: Physical, chemical and biological, respectively. The resulting working scheme is the basic rationale required to make any plan aimed to tackle the present challenge.

Then the impact is considered on solid Earth.

The next focus is on how electromagnetic (e.m.) induction phenomena originated by the Sun directly affect the deep interior of the Earth.

The impact is then briefly discussed on the ocean/atmosphere system, or on the biosphere, including extinctions.

A preliminary attempt is then made to consider some concrete actions that can be hopefully carried out.

A synthetic conclusion is finally outlined, including a focus (i) on fake news and mass media, (ii) on an unlucky conservatism of the scientific community that boycotts the exchange of truly innovative ideas, (iii) on the need for a multidisciplinary approach and (iv) on engineering perspectives for security.

Summarizing, an anomalous period is in progress, characterized by a very intense release of endogenous energy from the Earth. The cause is an ongoing heartbeat of the Earth's electrocardiogram. Heartbeats always occurred during the whole Earth's lifetime, at a 27.4 Ma pace. Humankind's history spans a much shorter duration. Hence, at present the humans are going to face an unprecedented challenge, maybe even concerning survival.

The duration of the climate anomaly of a heartbeat is a few Ma. Every heartbeat is typically characterized by two features: (i) The birth of a Large Igneous Province (LIP) and the present heartbeat is associated with the birth of Iceland and (ii) (often) by great extinctions. Life is endemic in matter. Microorganisms are steadily regenerated on deep ocean floors. The energy source is the exhalation of hot $\mathrm{CH}_{4}$. This is the beginning of the food-net that supplies lifeforms of increasing complexity. The same endemic phenomenon occurs on every object in the universe. The crucial control factor is a suitable environment and mostly the availability of some additional energy source, such as solar radiation. The appearance of more or less evolved lifeforms depends on survival and growth facilities. On the occasion of every heartbeat, some new lifeforms were eventually generated, while others experienced extinction. This will happen also during the present heartbeat. The challenge is to avoid that humankind is going toward extinction.

The paper attempts to give a general-and largely original and substantially advanced-perspective of Earth's phenomena. As far as possible, the approach is top-down, i.e., start from known laws and infer expected observations-in contrast with the more usual bottom-up viewpoint, i.e., start from observations and seek regularities.

The present text is devoted to applied scientists. The overall scenario is much more focused and precise than standard treatments. The target of the paper is concerned with the possible actions that can-and must-be implemented in order to face this unprecedented challenge-rather than being concerned with a critical discussion and comparison with the present "generally agreed" geophysical models reported in the literature. In particular, the exploitation is considered of a huge reservoir of natural and clean energy.

Humankind is a component of the natural system. Humankind is responsible for "pollution" that is evolving depending on human density and energy consumption. Pollution can be either negative or also positive when it contrasts some unwanted climatic trend. One key item is energy availability. The central focus of the present paper is the exploitation of the electrostatic energy of the atmospheric electrical circuit, which is a clean and almost inexhaustible free source.

It should be stressed that-as it has been proven by quantitative and precise observations-the $\mathrm{CO}_{2}$ pollution of anthropic origin provides an irrelevant-or maybe even a negligible-contribution to climate change. Climate change is, rather, caused by the natural, violent, ongoing exhalation of endogenous energy. This anomaly is going to be manifested, perhaps, by an eventual ever-increasing intensity of phenomena, including seismic and volcanic activity, energy and dynamics on the oceans and atmosphere, greater excursions with respect to the mild climate and a (presently unmonitored) change of endemic microorganism generation on the ocean floors, that, perhaps (?) in principle could even affect pandemics. 
This study is an attempt to synthesize-as far as possible and by a limited number of pages-a wide multidisciplinary scenario. The purpose is only to start a much more extensive and profound discussion, that-as far as the geophysical items are concerned-is the object of more extensive studies (in preparation).

\section{Drivers, Mechanisms and Methods}

\section{A Leading Endogenous Energy Source}

Tidal phenomena involve the entire Earth's system. The Moon is the main driver. The binary Earth-Moon system rotates around its barycenter. Two bulges are caused on the Earth (solid Earth, plus oceans, plus atmosphere): One bulge is directed towards the Moon, the other, owing to the centrifugal force, is antipodal (Fig. 1). This effect originates the well-known ocean tide, which is mainly a $\sim 12 \mathrm{~h}$ wave.

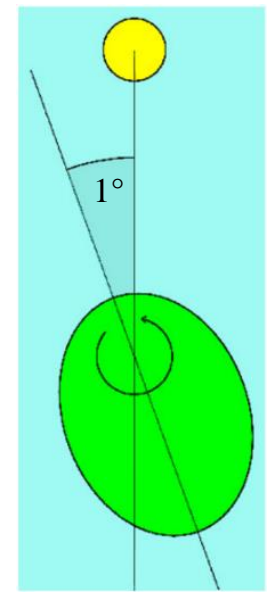

Fig. 1: Moon's tide. A double bulge is formed on the Earth. The Earth spins faster than the orbital motion of the Moon. Owing to the internal viscosity of the Earth, the Earth's swelling anticipates the motion of the Moon by $\sim 1^{\circ}$. The effect is an increase of both the length of the day (l.o.d.) and of the Moon's speed and distance from the Earth. The tide determines static and almost elastic deformation of solid Earth and dramatic dynamical effects on fluid circulation. Ocean tides pile up on the western coasts of oceans and determine the "loading tide" that causes the increase of the 1.o.d. The different component parts of the Earth's interior experience a comparably different tidal action. Hence, they experience relative displacements and being ionized, they represent a dynamo powered by the (lunar and solar) tide. This is called Tide-Driven (TD) dynamo, which is the main source of endogenous heat. See text. Unpublished figure

${ }^{3}$ The plane of the lunar orbit is inclined by $\sim 5^{\circ} .1$ with respect to the ecliptic plane.

${ }^{4}$ The consequent crustal effect can even be measured in the Mediterranean area.

${ }^{5}$ See Gregori (2002).

${ }^{6}$ Note the $10^{20} \mathrm{~J}$ and $10^{18} \mathrm{~J}$ values in the captions of Fig. 3
The spin of the Earth causes a rotation of the bulge (rotation is counterclockwise as seen from the Polar Star, i.e., eastward as seen at Earth's surface). The Moon moves on its orbit in the same direction, although with a period of $\sim 29$ days, i.e., at a much slower angular speed than the Earth. Thus, owing to the internal viscosity of the Earth, the Earth cannot reshape at a $24 \mathrm{~h}$ pace and the swelling anticipates the Moon by $\sim 1^{\circ}$ (Fig. 1).

The result is that the Moon slows down the spin rate of the Earth-thus causing the well-known increase of the length of the day (1.o.d.)-while, owing to action-reaction, the Moon's speed is increased while the Earth-Moon distance increases a by a few $\mathrm{cm}$ /year.

Solid Earth reacts to the tide, mainly like an elastic medium, unlike oceans that experience a large dynamic effect that affects global circulation. Thus, ocean waters pile-up on the western coasts of every ocean basin-the phenomenon is more pronounced at low latitudes due to the lunar orbit ${ }^{3}$. The most intense effect occurs in the Pacific Ocean resulting in water loading on the eastern coasts of Eurasia. The loading tide of the Atlantic Ocean pushes on the eastern coast of the Americas. The phenomenon is called "loading tide" and is the prime responsible for the observed increase of the 1.o.d. A huge stress is thus applied on the Earth's crust ${ }^{4}$ and it must slow down the whole planet-or at least it slows down the lithosphere, i.e., the outermost layer (a few ten to a few hundred kilometers thick) that corresponds to the layer involved by the drifting continents (Fig. 2 and section on Impacts-Solid Earth).

The Earth is a composite object. It is not a whole solid body-like, e.g., the Moon, which behaves almost like a solid glass-ball. Instead, different component-parts of the Earth can slide and drift relative to one another (Fig. 2). In addition, the tidal action is different on every internal component of the Earth, depending on the different distance from the Moon, hence on the local spatial gradient of the tidal force.

In general, every Earth's component has a relevant ionized fraction. Therefore, the tide supplies an effective dynamo-called Tide-Driven (TD) dynamo ${ }^{5}$. It is found ${ }^{6}$ that over $>99 \%$ of the generated energy decays like Joule heat and only $<1 \%$ is responsible for the geomagnetic field. The final energy balance ${ }^{7}$ is impressive (Table 1 and Fig. 3$)^{8}$.

${ }^{7}$ This refers, however, to the present period that is close to the peak of a heartbeat.

${ }^{8}$ The $T D$ dynamo mechanism also applies to other planetary objects. Consider the product of the diameter of the object times the space gradient of local gravitation. This is a significant physical index of the efficiency of the internal $T D$ dynamo. The Joule heat causes endogenous energy that originates tectonic activity. Hence, magnetic field and 


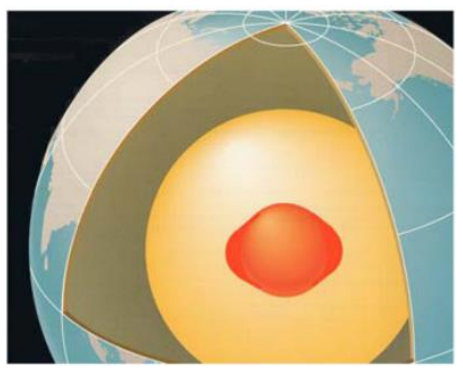

Fig. 2: The interior of the Earth (radius $R_{E} \sim 6370 \mathrm{~km}$ ) is characterized by a shallower (light brown) layer (lithosphere) of varying thickness (a few tens to a few hundred $\mathrm{km}$ ) that has the shallowest upper sub-layer (crust) that is of varying thickness (a few to several ten $\mathrm{km}$ ). The underlying (dark brown) layer (mantle) is extended down to $\sim 2900 \mathrm{~km}$ (mean depth). The underlying (yellow) layer is the Outer Core (OC), with an internal smaller object (inner core, or IC), that at present is $\sim 1250 \mathrm{~km}$ (mean radius, although not necessarily spherical). The OC is not crossed by seismic shear (S) waves. Hence, the OC is fluid, hot and highly ionized. Shear waves cross through the lithosphere, mantle-that are solid-and through IC, that cannot be solid. Matter in the IC is much more ionized than in the OC and nuclear magnetic moments are likely to play the dominant role, with strong inter-nuclear bonds that determine a structure that can be crossed by shear waves. The radius of the IC and maybe partly also of the OC, changes in time. Thus, the Earth performs like a battery that can charge and discharge at different times, storing energy by changes of state. See text. Unpublished figure

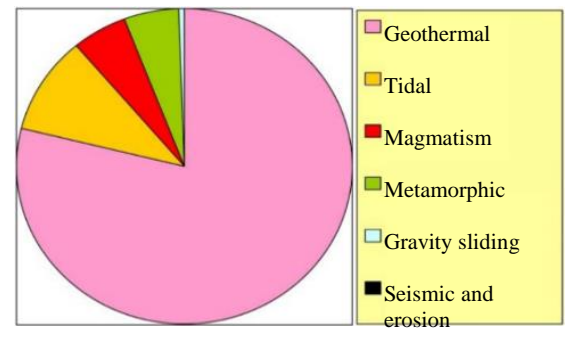

Fig. 3: Energy balance of the TD dynamo (Table 1). The total energy on either one of the three spherical shells (ss) is $10^{20}, 10^{18}, 10^{10} \mathrm{~J}$ for ICB, CMB, ALB, respectively. See text. After Gregori (2020). Reprinted with kind permission of Acoustics ("Open Access")
The Earth results to operate like a battery with cyclic discharge and recharge as follows. According to geomagnetic evidence (Fig. 4), the Earth's interior seems to contain 3 thin layers of currents-in fact, currents must expand as much as possible ${ }^{9}$ and concentrate and decay by Joule heat as soon as they meet a less conductive layer.

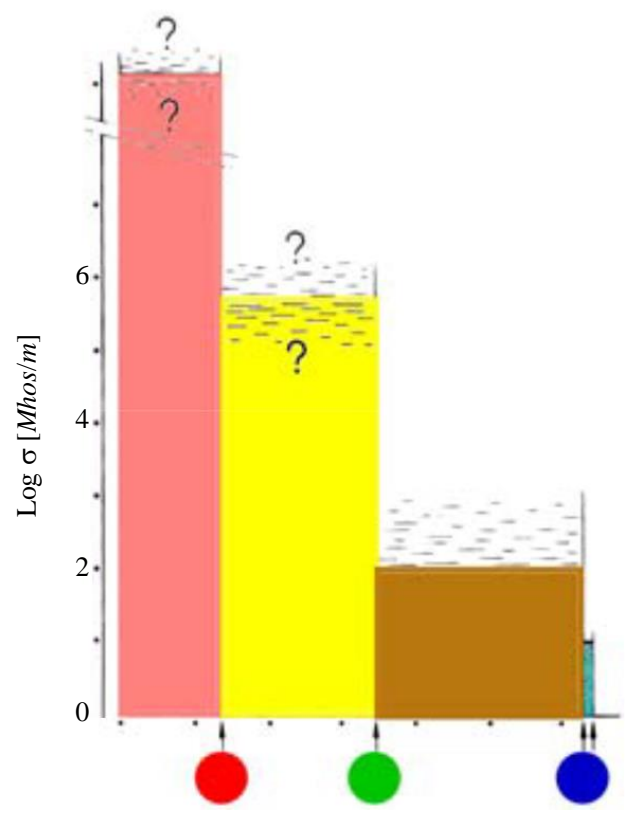

Fig. 4: The mean electrical conductivity $\sigma$ inside the Earth is characterized by three approximately step-like drops, respectively, at the transition between IC (pink), OC (yellow), mantle (brown) and lithosphere (light blue). Every loop of electric current $\mathbf{j}$ that flows inside the Earth must expand as much as possible until it reaches a less conducting layer, where the current decays by Joule heat. Thus, 3 layers of currents are generated, respectively, at the ICB (red), CMB (green) and ALB (dark blue). The three layers can be recognized by analyzing the energy of the geomagnetic field (Lowes-Nevanlinna law). The role of the IC ought to consider the peculiar state-of-matter, depending on nuclear magnetic moments. However, owing to a reasonable guess, this warning does not change the present approximate estimate. See text

Table 1: Endogenous energy of the Earth during the last few million years $\left(\times 10^{13} \mathrm{~W}\right)$

\begin{tabular}{llll}
\hline Kind of power & Estimate & Rough value & Percent \\
\hline Global heat flow measured across Earth's surface & $4.42 \pm 0.10$ & $\sim 4.42$ & $\sim 79$ \\
Tidal friction (total) & $0.4-0.7$ & $\sim 0.55$ & $\sim 10$ \\
Magmatic heat flux & $0.20-0.39$ & $\sim 0.3$ & $\sim 5$ \\
Metamorphic heat for transforming sedimentary and volcanic rocks & the same order of magnitude & $\sim 0.3$ & $\sim 5$ \\
Gravity sliding & $2.5 \pm 10^{-2}$ & $\sim 0.025$ & $\sim 0.5$ \\
Seismic S & $0.3-3 \pm 10^{-3}$ & $\sim 0.5$ \\
Potential energy loss due to soil erosion & $3 \pm 10^{-4}$ & $\sim 5.56$ & $\sim 0.0$ \\
Total & & 100 \\
\hline
\end{tabular}

tectonic activity must be correlated. All available observational evidence confirms this correlation.
${ }^{9}$ Refer to college physics, or to the Hamilton's variation principle. 
Owing to energy constraint, every current sheet of the 3 thin $\mathbf{j}$-layers must have a mean radius smaller than a given asymptote. The 3 asymptotes surprisingly result to be only by a few per cent larger than the seismically determined radii, respectively, of the Inner Core Boundary (ICB), of the Core-Mantle Boundary (CMB) and of the Asthenosphere-Lithosphere Boundary (ALB). The 3 layers are not spherical, although, upon referring to their mean radius, they can be approximately indicated by 3 spherical shells (ss). Hence, they are briefly called, respectively, $s s_{l C B}, s s_{l M B}$ and $s s_{A L B}$, even though in general they are not spherical.

Differently stated, the mean radii of ICB, CMB and ALB can be determined either by the classical seismological method, or (with an excess of only a few percent) by means of the classical geomagnetic Secular Variation (SV). Suitable models of the SV are available since the middle of the XV century AD. It is thus found that the Earth battery stores or releases energy by changing-even by some large amount-mostly the ICB radius ${ }^{10}$ and, to a lesser extent, also the CMB radius.

An external modulation of the TD dynamo is operative.

In fact, interplanetary space is not vacuum. Rather, it is filled-up with the expanding solar corona, that constitutes the "solar wind", that transports a "frozen-in" Interplanetary Magnetic Field (IMF or $\mathbf{B}_{\text {int }}$ ) that lies in the ecliptic plane (apart lesser fluctuation of, say, $\sim 1-2^{\circ}$ ).

The IMF is oriented according to the well-known Parker spiral, i.e., at Earth's orbit it is directed at $\sim 45^{\circ}$ with respect to the Earth-Sun line, being oriented sometimes toward, some away, relative to the $\mathrm{Sun}^{11}$.

The Earth's is protected by a magnetic shield that forbids direct interaction and spoiling of the atmosphere by the solar wind. The shield is wellknown, being the magnetosphere, that results from the e.m. interaction of the solar wind with the geomagnetic field $\mathbf{B}$. The shield opposes the penetration of the solar wind (mainly electrons, protons and ions). Additional details are given in section on The MOR Network, the Deep Tetrahedron/OQR Pattern and Sun-Earth Relations (Figs. 22 and 23).

A mention is deserved for the analogous phenomenon that involves the whole Solar System, when it encounters the interstellar wind, i.e., clouds of interstellar matter. "Heliosphere" is called a shield of the whole Solar System, that is analogous to the Earth's magnetosphere.

The matter density of a cloud of interstellar wind ${ }^{12}$ can vary by conspicuous amounts.

\footnotetext{
${ }^{10}$ Seismic evidence confirms the trend vs. time of the ICB radius on the time scale of a few decades, consistently with the trend provided by the geomagnetic SV since the middle of the XV century.

${ }^{11}$ The reader should be warned about an erroneous information reported by several authors, who consider the IMF as being
}

Thus, occasionally, the heliosphere can be compressed very close to the Sun, even inside the Earth's orbit. On every such an occasion, the Earth experiences an abrupt "disappearance" of the solar wind.

The effect is devastating on the TD dynamo of the Earth. The geomagnetic field $\mathbf{B}$ can eventually fade off, either entirely or partially.

But shortly afterwards the solar wind reappears. During this whole process (lasting a few thousand years), huge e.m. induction currents are generated inside the deep Earth's interior. The TD dynamo is violently reactivated. But, owing to a well-known effect (e.g., similarly to a household electric juicer), there is a $50 \%$ probability that the newly-born geomagnetic $\mathbf{B}$ has the same orientation as the older field and 50\% probability that it is reversed. These phenomena are the well-known geomagnetic Field Reversals (FRs) that occurred a huge number of times during Earth's history. FRs display an erratic time sequence, depending only on the location of interstellar clouds.

It should be stressed that a huge number of incomplete "reversal" events are also observed and in general are called "geomagnetic excursions", although a warning deals with the spatial extension of the region where the associated geomagnetic anomaly is observed. In fact, one cannot exclude that they are originated by "local large" electric currents, or sporadic intense discharges. Indeed, this justifies the need for investigation of the unexploited realm of anomalous electric phenomena, which is one leading focus of the present paper.

FRs have a tremendous impact on climate. A huge amount of Joule heat is generated, that affects soil exhalation, geodynamics, volcanism and atmospheric density. In addition, in general, several FRs occur during the large energy-release anomaly of a heartbeat, hence it is impossible to exploit any direct correlation with FRs data series that act indirectly. At present, we are in a longlasting period (>700.000 years) with no FR.

Also a large impact occurs on atmospheric density. In fact, soil exhalation increases the mass of the atmosphere, that-in contrast-decreases during the disappearance of the geomagnetic field and of the magnetosphere. Consider that, on the occasion of a FR, during a few thousand years the shield-magnetosphere disappears and the spoiling is operative by a timevarying solar wind. Thus large variations occurred in the past of both atmospheric density and composition (see section on The MOR Network, the Deep Tetrahedron/OQR Pattern and Sun-Earth Relations).

perpendicular to the ecliptic plane. This false belief was originated by a tentative and incorrect working hypothesis. But the observational evidence is very clear and rebuts such an assumption. ${ }^{12}$ The monitoring of interstellar clouds is carried out by radio telescopes. 


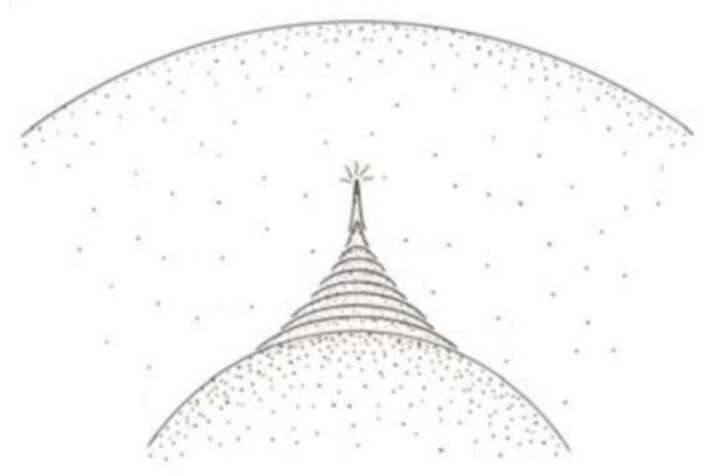

Fig. 5: Owing to Hamilton's principle, a former tiny bumpwith respect to a perfect spherical surface with electric currents $\mathbf{j}$ flowing on it-is the site of a comparably more intense $\mathbf{j}$ concentration, where local temperature and electrical conductivity $\sigma$ are increased. Thus, the bump penetrates outward and sharpens, generating a sea-urchin spike. The whole mechanism looks like an electric soldering iron that penetrates through ice, hence the name ESI mechanism. See text. Reproduced with kind permission of the late Wilfried Schröder. Also in (Gregori, 2020)

Summarizing, the pace of the electrocardiogram is determined by the internal structure of the Earth (charge and discharge of the battery). A superposed $\sim 14 \mathrm{Ma}$ periodic modulation is also observed, which is of external origin and is likely to be associated to the oscillation of the Solar System with respect to the equatorial plane of the Galaxy. This same mechanism ought to contribute to refill the Solar System reservoir with meteorites and comets (Oort reservoir).

Altogether the "climate" control occurs therefore through a chain of Galaxy-Sun-Earth relations.

\section{The Propagation up to Earth's Surface and to the Atmosphere}

According to college physics, electric circuits expand in space as much as possible (by the Hamilton's variation principle). Owing to Hamilton's, if a lesser and gentle bump occurs on either one of the 3 ss, the electric currents must concentrate on the top of the bump, where they release Joule heat. Therefore, the local temperature increases and causes an increase of the local electrical conductivity $\sigma$. Thus, the former lesser bump progressively penetrates upward, while it sharpens (Fig. 5), thus reminding about the

\footnotetext{
${ }^{13}$ An older and equivalent, term for superswell is megaundation, or-on a smaller size - geotumor (van Bemmelen).

${ }^{14}$ Due to the errors related to the difficulty to collect samples of deep ocean floors.

${ }^{15}$ Several chemical reactions have been envisaged, mainly involving compounds of Mg. See Judd and Hovland (2007) for details.
}

penetration of an Electric Soldering Iron (ESI) that is pushed into a block of ice-hence the name ESI mechanism. The final result is that-in terms of electric currents-the Earth's interior looks like being crossed by spikes, reminding about a seaurchin.

Different amounts, of sea-urchin spikes, cross through different regions of the Earth and originate a different thermal expansion of the mantle, that determines, therefore, a more or less large uplift of the lithosphere. Thus, huge uplifts occur at Earth's surface, almost like gigantic "hills", that are called superswells ${ }^{13}$.

The present superswells can be significantly indicated in Fig. 6. The Mid-Ocean Ridges (MORs) are located on top of superswells. When a superswell is uplifted, the overlying lithosphere slides down on the surrounding slopes. The outpoured material embeds the record of the direction of the geomagnetic field $\mathbf{B}$, much like it happens for the lava effusion of every other volcano. Thus, the well-known stripes of geomagnetic anomalies, that are observed to lie parallel to the respective MOR, represent the FRs time series-although the best dating ${ }^{14}$ is achieved by studying land sediments.

Water is a very simple molecule, ubiquitous in the universe. Water plays a fundamental role in the Earth. A reasonable inference is that a shallower Earth's layer contains a large water concentration, while the layer underneath is comparatively less hydrated. Thus, at the transition between the more and less hydrated layers, the speed Vs of the shear (S) seismic waves abruptly increases from $\sim 3.7$ to $\sim 4.3 \mathrm{~km} \mathrm{sec}^{-1}$. This is the classical boundary known as Moho, at a typical depth in continental areas of $25-60 \mathrm{~km}$ and of 5-8 $\mathrm{km}$ beneath ocean floors.

Water, however, also penetrates beneath the Moho, where it experiences a chemical and explosive interaction with dry rocks (serpentinization ${ }^{15}$, cfr. the chemical engine in section Synthesis. Three Engines: Physical, Chemical, Biological). Rocks are cracked by the resulting explosion and water can further penetrate. Thus, an almost ubiquitous thick serpentinized layer is formed all over the Earth. The layer is named "serpentosphere", composed of fractured and hydrated rocks, embedded inside water that, owing to the high pressure, has peculiar properties and it is termed "supercritical water" (Judd and Hovland, 2007) ${ }^{16}$.

\footnotetext{
${ }^{16}$ Supercritical water occurs at a pressure $>220$ bar. It is heated, although it cannot boil. Boiling stops at $374.15^{\circ} \mathrm{C}$, at a highest pressure of 221.2 bar (for pure water without salts). The bulk density is $0.322 \mathrm{~g} \mathrm{~cm}^{-3}$ with specific volume 0.31 . Supercritical water is no longer dipolar. $\mathrm{pH}$ varies in the range $8 \leq \mathrm{pH} \leq 12.7$. See Judd and Hovland (2007) for details.
} 


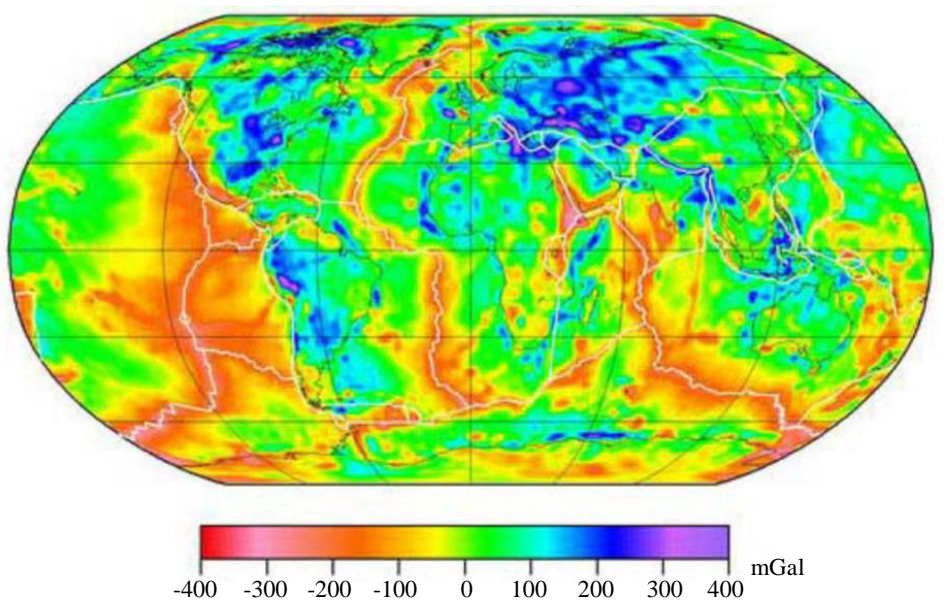

Fig. 6: Gravity anomalies according to the GRACE model after subtracting the contribution by matter above the Moho. MORs show the present location of superswells, while a huge megasyncline runs from the Pyrenees through Japan. See text. Credit:http://www-app2:gfz-potsdam:de/pb1/op/grace/results/index_RESULTS.html; gravity field model EIGEN-GRACE01S released on Jul 25, 2003. With kind permission of GFZ Deutsches GeoForschungs Zentrum

The serpentosphere is characterized by high electrical conductivity $\sigma$ and by a low acoustic impedance (i.e., by an efficient ultrasound propagation). Hence, owing to Hamilton's, all electric currents in the mantle must expand on the outermost boundary of the highly conducting serpentosphere. This boundary coincides with the ALB. The ALB is therefore a highly lubricated layer, being a mixture of fractured rock and supercritical water. Lubrication is eventually greatly improved (Fig. 9) by the Joule heat of the currents that leak off the CMB (leakage involves a $\sim 10^{-8}$ amount of CMB currents; see captions of Fig. 3).

As far as depth and thickness are considered, the ALB is the lower surface of the lithosphere, that, according to seismological and geothermal evidence, ranges between a few $10 \mathrm{~km}$ in oceanic areas (or in the Tyrrhenian Sea) through $>400 \mathrm{~km}$ in Africa. The serpentosphere is a layer beneath the ALB, of unknown and varying thickness, identified with some upper layer of the mantle ${ }^{17}$.

The final result is that the lithosphere can easily slide on the ALB, downslope of superswells and piles up into huge megasynclines, where thrusting-and eventually also some folding-occur, generating mountains and continents. At present, a huge megasyncline runs from the Pyrenees through the Himalaya and it is extended until Japan.

\footnotetext{
${ }^{17}$ In some respect, perhaps, the serpentosphere can be (improperly) identified with the concept that is often intuitively called "asthenosphere" being a low viscosity layer.

${ }^{18}$ The spelling Hawai'I is here adopted instead of Hawaii, as it seems to be, maybe, better consistent with the historical origin of the name.
}

Unlike the well-known plate tectonic model-which is proven to be inconsistent with observations-this mechanism based on superswells looks like in the case of some kind of "warm mud" that slides on the ALB. Thus, this model is called "Warm Mud Tectonics" (WMT).

On the other hand, continents are rapidly destroyed by weathering and erosion. The cycle of continent generation and destruction is of $\sim 100-200 \mathrm{Ma}$ (million years), maybe of $\sim 180 \mathrm{Ma}$ (Mortari cycle).

Owing to several concurring evidences, two large regions, antipodal to each other, are found to manifest a particularly intense exhalation of endogenous heat. The most intense phenomenon is displayed as-and it is generally identified with-the so-called Hawai'ian hotpot $^{18}$. The antipodal wide region is around Botswana. Among other evidences, a relevant peculiarity is manifested by the isotopic chemism of ocean basalt-that, perhaps, is associated also with the formation of diamond-bearing kimberlites ${ }^{19}$ (Botswana is the greatest producer of diamonds).

Volcanism is a comparatively rare and exceptional phenomenon (Fig. 3), although it is the spectacular Earth's surface signature of the sea-urchin spike pattern ${ }^{20}$. As mentioned above, a sea-urchin spike penetrates upward by the ESI mechanism. Whenever the top of the spike gets close to Earth's surface, the available fluids (water and geogas ${ }^{21}$ ) transport heat by advection. If fluids

\footnotetext{
${ }^{19}$ Diamond formation is the object of a huge literature. The present mention is only aimed to point out the "coincidence" of diamond bearing kimberlites with specific geodynamic and tectonic features.

${ }^{20}$ Note, however, that a large fraction of volcanoes is supplied by friction heat (see below).

${ }^{21}$ Geogas is a general term including all kinds of gas that can exhale from the Earth.
} 
are insufficient, the local temperature increases. But, owing to the reduced lithostatic pressure (i.e., the weigh exerted by the overlaying layers), the equation-of-state permits melt and magma is formed, much like a specifically devoted new fluid. Finally, lava effusion transports heat by advection ${ }^{22}$.

A volcano operates like the security valve of a pressure cooker. Endogenous heat is accumulated. When the volcanic edifice is insufficient to plug the endogenous pressure, the volcano erupts. Volcanoes that erupt lava are the least hazardous case histories. In contrast, the most catastrophic events occur when the plugging is very efficient and the volcano can only explode to produce an exceptionally violent catastrophe.

Note, however, that volcanoes can be supplied either by a sea-urchin spike, or by local friction-heat. For instance, concerning the largest percent of volcanoes that lie along the so-called ring of fire of the Pacific Ocean, they are originated by friction-heat deriving from WMT.

A detailed mechanism occurs that leads to the typical morphology of the so-called island $\operatorname{arcs}^{23}$.

According to a recent finding ${ }^{24}$ (Quinn, 2014), huge electric air-earth currents occur between soil and atmosphere $^{25}$, up to the highest atmosphere. The phenomenon is associated with soil exhalation from heavily fractured crust. It is now well-known that phenomena in the ionosphere (and magnetosphere) are associated with earthquakes (precursors, co-seismic events, aftershocks).

The hotspot concept is mentioned in a large literature, although no agreed definition is available. In general, a hotspot is speculated to be caused by some "plume" that ought to exist somewhere, slowly rising from the deep Earth's interior. The concept of the very rapid electric transport (i.e., only electron motion occurs) by sea-urchin spikes is a substitute, that is strongly supported by observations $^{26}$. In any case-since the XV century AD and

\footnotetext{
${ }^{22}$ Note the nonsensical fashionable assumption that lava circulates underground like inside the plumber network for an aqueduct. Such a model is totally unstainable according to several physical reasons.

${ }^{23}$ The role of friction heat was clearly focused by the analysis of the Hawai'ian hotspot that led to the proof of the electrocardiogram (Figs. 7 to 9). The absence of any geomagnetic signature associated with island arc volcanism shows that friction heat is the dominating driver, while every magnetic implication is just accessorial.

${ }^{24}$ John M. Quinn passed away on March 31st, 2020. We lost a friend, the scientific community lost a great scientist.

${ }^{25}$ This result is in contrast with the old-fashioned and never checked classical working hypothesis by Gauss, who assumed that the "mean" effect of air-earth currents can be neglected. This hypothesis permitted the exploitation of the well-known classical algorithm suited to separate the geomagnetic field of internal and of external origin. The Quinn's finding is, maybe, the greatest "revolution" in geomagnetism reported since the time of Gauss.
}

according to geomagnetic evidence-just 30 and only 30 , hotspots have been active and every hotspot was associated with a huge local volcanic activity that, however, later eventually continued, although in general with no equivalent comparably violent paroxysm. This feature is suggestive that, after the first removal of the "plug", the hotspot no more affords to fully recover and reset the former energy reservoir ${ }^{27}$.

The best-known hotspot is Hawai'ian. The model is in terms of the lithosphere that moves like a conveyor belt on top of an endogenous heat source (Fig. 7). Thus, the Hawai'i-Emperor Seamount Chain is composed of 108 volcanic edifices, the largest part of which is submerged.

Therefore, according to a classical Tuzo Wilson's model (Fig. 8), it is possible to estimate the intensity of the endogenous source during the last $\sim 70 \mathrm{Ma}$, on the basis (i) of the volume of the 108 volcanic edifices, combined with (ii) the date of the earliest eruption that constructed every volcanic edifice ${ }^{28}$. The result is shown in Fig. 9, where it should be noted that the drift velocity is more reliable than the volume of outpoured magma. In fact, the volume of outpoured magma is affected by the difficulty to estimate the volumes of overlapping volcanic edifices.

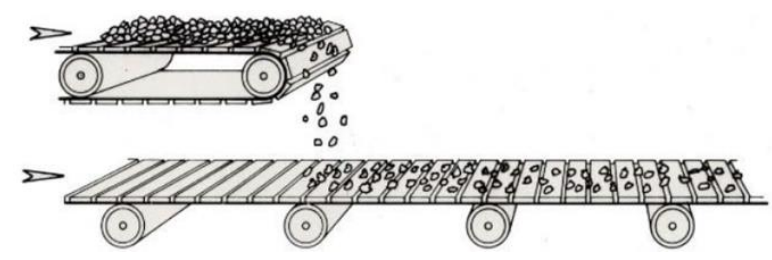

Fig. 7: Conceptual model to simulate magma outpouring from a hotspot. An upper conveyor belt, which simulates the hotspot output, transports sand that drops over a lower conveyor belt, that moves at some unknown, eventually time varying, speed. See text. After Gregori (2002). With kind permission of the late Wilfried Schröder

\footnotetext{
${ }^{26}$ Note that the "plumes" neglect the role of energy transport by electric currents. In this respect, also note that the frequently mentioned and speculated "mantle convection" never found observational support. Hence, for the time being, the "mantle convection" concept is only speculative and must be neglected or rebutted.

${ }^{27} \mathrm{~A}$ conspicuous literature exists dealing with hotspot definition and also with a speculated hotspot framework and with the relative drift between hotspots. No final evidence and agreement were ever attained, not even on the total number of hotspots. On the other hand, in the section on The MOR Network, The Deep Tetrahedron/OQR pattern and Sun-Earth Relations it is shown that all Earth's surface signatures, including the speculated hotspots, are deformed remnants of the exact deep tetrahedron pattern. Hence, all discussions about hotspot reference frame and hotspot drift are to be considered an irrelevant or meaningless concern.

${ }^{28}$ See detail in Gregori (2002).
} 


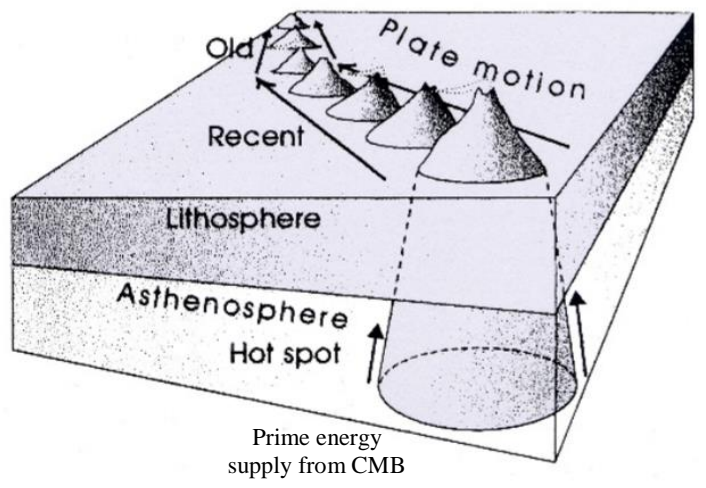

Fig. 8: The chain of the Hawai'ian islands and of the Emperor Seamounts is composed of 108 elements (i.e., islands or seamounts) of increasing age in sequence from Kîlauea towards Kamchatka. Their classical (Tuzo Wilson's) interpretation is in terms of a movement of the lithosphere, sliding over the asthenosphere, much like a conveyor belt that collects the magma outpoured from a fixed hotspot. Upon measurement of the volume of the elements of the chain and of their age, it is possible to monitor both the time variation of the magma emplacement rate from the hotspot and also the speed of the drift of the lithosphere. Owing to the behaviour of a volcanic edifice like the security valve of a pressure cooker, a sequence of discrete single elements is observed, instead of a continuous belt of outpoured magma. The data interpolation is based on the measured age and location of the elements. In principle, a similar and different computation of the same pattern can also be carried out (although not available) based on the estimate of the age of the ocean floor over which magma is emplaced, evaluated by the timing of the paleomagnetic stripes of the ocean floor. After Gregori (2002). With kind permission of the late Wilfried Schröder
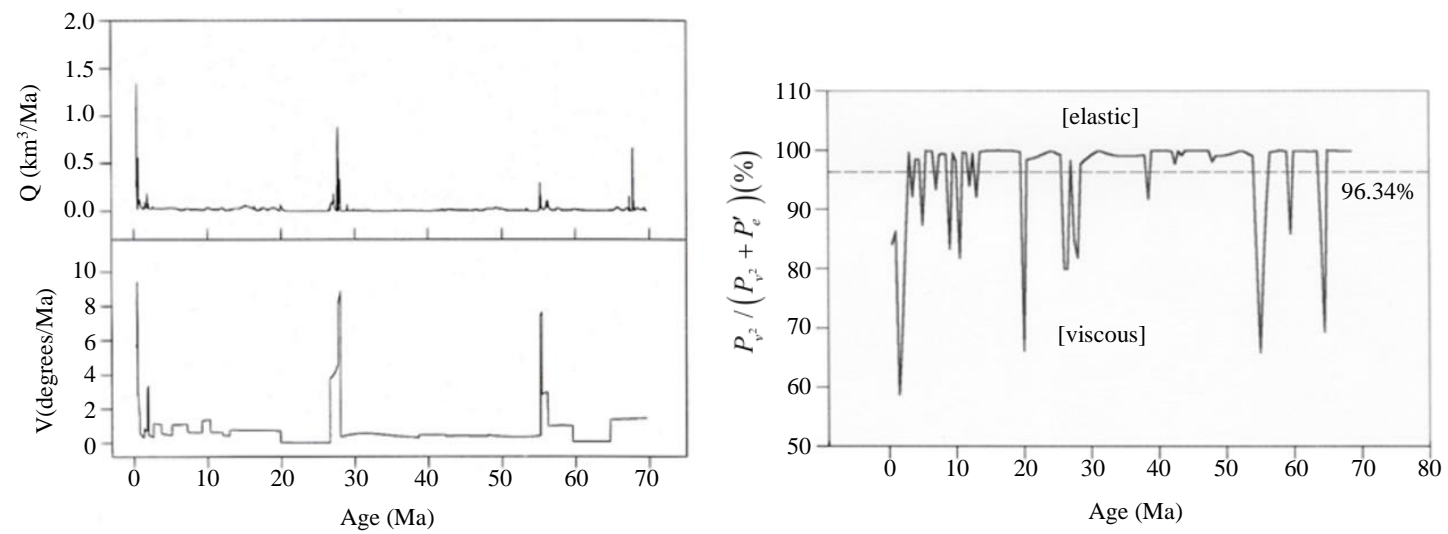

Fig. 9: [left plot] Electrocardiogram of the Earth-or magma emplacement rate per unit time from the Hawai'i hotspot during the last $\sim 70 \mathrm{Ma}$, derived by formal interpolation over the volume of the outpoured islands and seamounts of the Hawai'i-Emperor Seamount chain vs. their respective age. The resolution time is 100,000 years. One heartbeat occurred every $\sim 27.4 \pm 0.05 \mathrm{Ma}$, a trend that seems to have been, maybe, a steady feature during the last $\sim 250$ Ma. Every heartbeat corresponds to the emplacement of either one or a few LIPs. The LIP corresponding to the current heartbeat is likely to be Iceland. The two diagrams in the left plot show, respectively top to bottom, the volume of magma emplacement and the speed of the lithosphere relative to the hotspot source. Note that the speed estimate is likely to be more reliable, as it is less affected by the errors in the computation of the volume of every volcanic edifice. [right plot] Inspecting the relative contribution, vs. time, of the viscous vs. the elastic component in the coupling between lithosphere and mantle (the mean is $96.34 \%$ viscous and $3.66 \%$ elastic coupling), with huge time variations depending on heartbeat pace. The estimated errorbars can be of the same order of magnitude as the effect that is to be detected. In any case, the result is fairly consistent with expectation. In addition, the identical inference is derived by the same database treated by two different criteria for data handling. The large errorbars depend on the approximations either of the method of analysis, or of the available raw observational database. See text. After (Gregori and Dong (1996; Gregori, 2002)

The maximum detected speed is impressive, being as large as the order to $\sim 3 \mathrm{~mm} \mathrm{day}^{-1}$. Fig. 9 is called Earth's electrocardiogram, that displays a pace of $\sim 27.4 \pm 0.05 \mathrm{Ma}$. Every heartbeat lasts 4-5 Ma. The electrocardiogram can be tracked - by allowing for some error-bar-through $\sim 250 \mathrm{Ma}$ or even longer. Note that, during the short time-lag of every large release of endogenous energy, the coupling at ALB is mainly viscous (i.e., the ALB is much lubricated), unlike during other times when the coupling is mainly elastic (i.e., the ALB is poorly lubricated). The difference is impressive, 
it depends on the temperature at the ALB and the result is the same even when different computational criteria are assumed (see Gregori, 2002, for details).

The electrocardiogram mechanism is as follows. Sea-urchin spikes spend $\sim 27.4 \mathrm{Ma}$ to cross from the CMB through the mantle (at a mean speed of $\sim 10 \mathrm{~cm}$ year $^{-1}$ ). As soon as the spikes approach a sufficiently shallow layer, a fluid (also magma, when melt occurs) transfers endogenous heat by advection up to the ocean/atmosphere. In the meantime, superswells are uplifted, geodynamics, seismic and volcanic activity intensified, while climate is severely affected, including (maybe) also endemic life generation.

As soon as an efficient "channel" is opened through which endogenous energy can be released, the internal reservoir is progressively discharged, while the TD dynamo is incapable to fully refill the energy loss. The Earth's interior rapidly cools off, the electrical conductivity $\sigma$ decreases, the spikes are no more supplied by flowing current and are therefore "cutoff" at their base. Thus, the TD dynamo can restart to charge the internal reservoir. The battery stores energy through the observed change of the radii of ICB and CMB.

On the occasion of every heartbeat, a huge magma release occurs somewhere at Earth's surface, leading to the birth of a large igneous province (LIP), which looks like a tremendous lava-flood emplaced during a few million years.

At present, the Earth is at the top of a heartbeat and the associated LIP is Iceland. In fact, Iceland $\sim 2 \mathrm{Ma}$ ago did not exist ${ }^{29}$. That is, at present the Earth is during the "Iceland heartbeat".

Also, the heating at the ALB experiences a huge cyclic variation, depending on the timing reckoned after the start of the last previous heartbeat. The phenomenon is documented by the ratio of the plastic/elastic coupling between lithosphere and asthenosphere (Fig. 9): The colder the ALB, the less the melt component and lubrication and the more elastic the coupling.

A strong modulation-by the number and timedistribution of the FRs that occurred in the time-lag between successive heartbeats-is documented in the detailed analysis (Gregori, 2002) of the data set of Fig. 9. It is thus possible to assess that an increase of the released endogenous heat produces an increase of the kinetic energy of the drift velocity of the lithosphere and only after some $\sim 50,000$ year friction-heat originates lava effusion.

In any case, the leading component of the global energy balance is represented by the geothermal flow, as per Fig. 3, that results to be the leading driver for climate. That is, the prime effect of the ongoing climatic change derives from the time-change of the release of unnoticed geothermal heat on the planetary scale.

\section{Synthesis. Three Engines: Physical, Chemical, Biological}

The whole set of drivers and processes that control the evolution of the Earth can be synthesized in terms of three engines (Fig. 10).

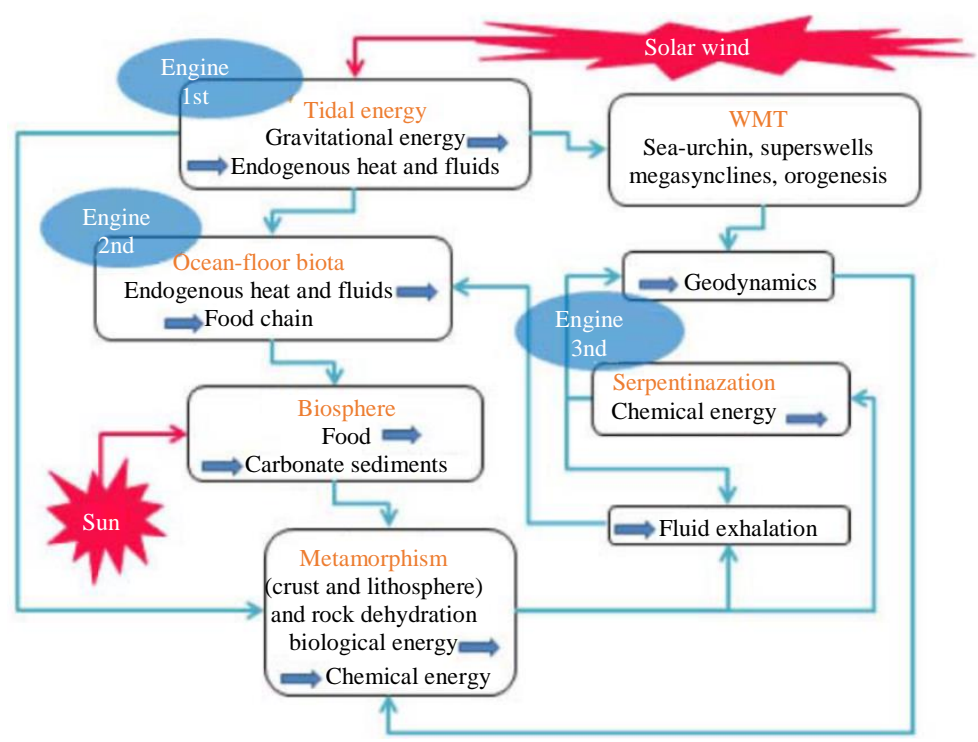

Fig. 10: The leading drivers of "climate" are three engines. The Sun, through the solar wind, modulates the physical engine (i.e., the $T D$ dynamo, the main energy source being the lunar tide). In addition, solar irradiance amplifies the role of the food chain originated by fluids and energy exhalation from soil. The generated biomass finally returns into ground in the form of organic sediments. After Gregori (2015; 2020). With kind permission of New Concepts in Global Tectonics

${ }^{29}$ Also, some older rocks are found in Iceland, that are a signature of the previously existing and uplifted crust. The older non-existence of Iceland is testified by the magnetic lineaments of the geomagnetic anomalies of the Atlantic Ocean floor, according to a reconstruction made by unpublished data on geomagnetic anomalies provided by oil companies (Helman et al., 2001). 
The physical engine is the TD dynamo, supplied by the tide, which is mostly of lunar (and by a lesser amount solar) origin. The efficiency of the TD dynamo is modulated by the e.m. induction from the solar wind. The overwhelming output is represented (Fig. 3) by thermal energy of Joule heat, manifested in different ways. But the direct flow of endogenous energy is essential in a few respects. On the one hand, it is responsible for the dehydration of rocks. Dehydration is strictly needed to overcome the hydration caused by serpentinization. On the other hand, direct flow of endogenous energy is responsible for the steady endemic regeneration of life on the ocean floors (see below the biological engine) and-in addition-it is also a leading driver for climate change.

The chemical engine is serpentinization that generates the serpentosphere (concerning depth and thickness, see section on The Propagation up to Earth's Surface and to the Atmosphere), that plays a crucial role for producing a likely ubiquitous layer (the asthenosphere, hence the ALB) composed of fractured rocks and of supercritical water.

One consequence is the lubrication that permits an easier sliding of the lithosphere. The sliding is a steady persistent phenomenon, eventually with large variations of flow speed according to Fig. 9. In any case, the lithospheric sliding is fundamental for geodynamics and seismicity. In addition, the lubrication effect is further amplified by the electric currents, caused by the TD dynamo, that leak off from the CMB (roughly by a fraction $\sim 10^{-8}$; see the captions of Fig. 3) and propagate up to upper layer of the serpentosphere, where they supply heat to the ALB. The amount of this amplification-hence also seismicity and volcanism-changes in time during the time interval between two heartbeats (Fig. 9).

Another fundamental role of the serpentosphere is the very low acoustic impedance, by which ultrasound can propagate on the planetary scale. This fact results to be a great facility for diagnosing the state and evolution of crustal stress (for seismic prediction; Gregori et al., 2018).

Serpentinization occurs only if rocks are dehydrated, but the physical engine ensures dehydration of previously hydrated rocks.

The biological engine is represented by the endemic generation of life inside matter. Biological processes flourish only whenever suitable environmental condition permits life generation and survival. Thus,

\footnotetext{
${ }^{30}$ Martin Hovland is to be acknowledged for fundamental contributions in the assessment and mapping of $\mathrm{CH}_{4}$ exhalation on the planetary scale, for the endemic character of life regeneration on the deep ocean floors and for the formulation of the concept of
}

microorganisms are presently steadily regenerated on the very deep ocean floors, with the energy supply from hot geogas, mostly $\mathrm{CH} 4$ and $\mathrm{CO} 2$, that constitute the primary fuel for life (Judd and Hovland, 2007)30. This is the beginning of the food web, that supplies other living organisms of increasingly evolved species, that occupy progressively shallower ocean layers. When solar radiation becomes the leading energy source, highly evolved living forms can be generated, that eventually occupy even emerged land and the atmosphere.

Note that, in general, new forms of microorganisms are permanently generated everywhere (viruses, microorganisms, etc.), depending on the varying environment that changes with the time-varying release of endogenous energy. Hence, one must expect a steady uninterrupted sequence of generation of unprecedented forms of life, while other forms experience extinction-as, indeed, it is documented by paleontology (see section on Impacts-Solid Earth).

Thus, the "principle of preservation of life" has been formulated (Ronov, 1982; Budyko et al., 1987 and Fig. 11), i.e., with no soil exhalation (that can occur either in "quiet" form, or through various forms of volcanism) life would soon become extinct.

In fact, this leads to explain the carbon cycle (Fig. 12). Carbon is exhaled from the Earth's interior through $\mathrm{CH}_{4}$ and $\mathrm{CO}_{2}$. A one-year record and mapping of atmospheric concentration of $\mathrm{CO}_{2}$ is available ${ }^{31}$ that, however, proves that the $\mathrm{CO}_{2}$ of anthropic origin plays an irrelevant-or maybe even a negligible-role (Gregori et al., 2018; Gregori, 2020). In contrast, no equivalent planetary monitoring is yet available concerning $\mathrm{CH}_{4}$.

Life evolves from more elementary forms (such as corals) through the whole realm of plant and animal world. Carbon is finally transformed into sediments and deposited in sterile carbonate rocks on the ocean floors.

These rocks, are dehydrated by endogenous heat and are needed for the chemical engine, i.e., for serpentinization, that generates hydrated rocks that are no more suited for serpentinization, but the endogenous heat produced by the physical engine determines the dehydration of hydrated rocks. Dehydrated rocks are suited for serpentinization, etc. Thus, the cycle is completed and the "principle of preservation of life" can be operative.

Note that the same principle applies to every object in the Solar System-and in the universe. In fact, life ought to be endemic everywhere. The first requirement is that matter is sufficiently cold in order that chemistry

serpentosphere. Certainly, he is one of the most important living scientists who contributed to a substantial progress in Earth sciences.

${ }^{31}$ Monitored by the NASA's OCO-2 satellite (Mearns, 2015a; 2015b). 
can be operative (in fact, the environment is unsuited for chemistry and biology when it is heavily ionized such as inside a star). The degree of subsequent evolution of the living forms depends only on the environmental conditions.

For instance, life is possible on several planetary objects, although it must be expected to occur eventually only underground, as solar radiation is generally insufficient to supply a significant amount of energy. That is, one must expect that, underneath the surface of several planetary objects, a fluid layer is eventually found, due to the endogenous heat originated by an internal TD dynamo. The fluid composition is likely to contain a large percent of $\mathrm{H}_{2} \mathrm{O}$, which is one of the most elementary molecules in the universe. Life, however, cannot survive above the surface of the planetary object, as the Sun is excessively far away. Indeed, this is the reason by which the exploration of the Solar System is searching for proofs of the underground existence of life, in the form of microorganisms.

In this same respect, a search for large-size lifeforms in the Mariana Trench near Guam, at a depth of $\sim 8,000 \mathrm{~m}$, captured snailfishes in the length-range 89$235 \mathrm{~mm}$ (Gerringer et al., 2017). These life forms cannot migrate to shallower levels due to very large pressures and they flourish with no contribution from solar radiation. Therefore, maybe, similar life can also be found on other planetary objects.

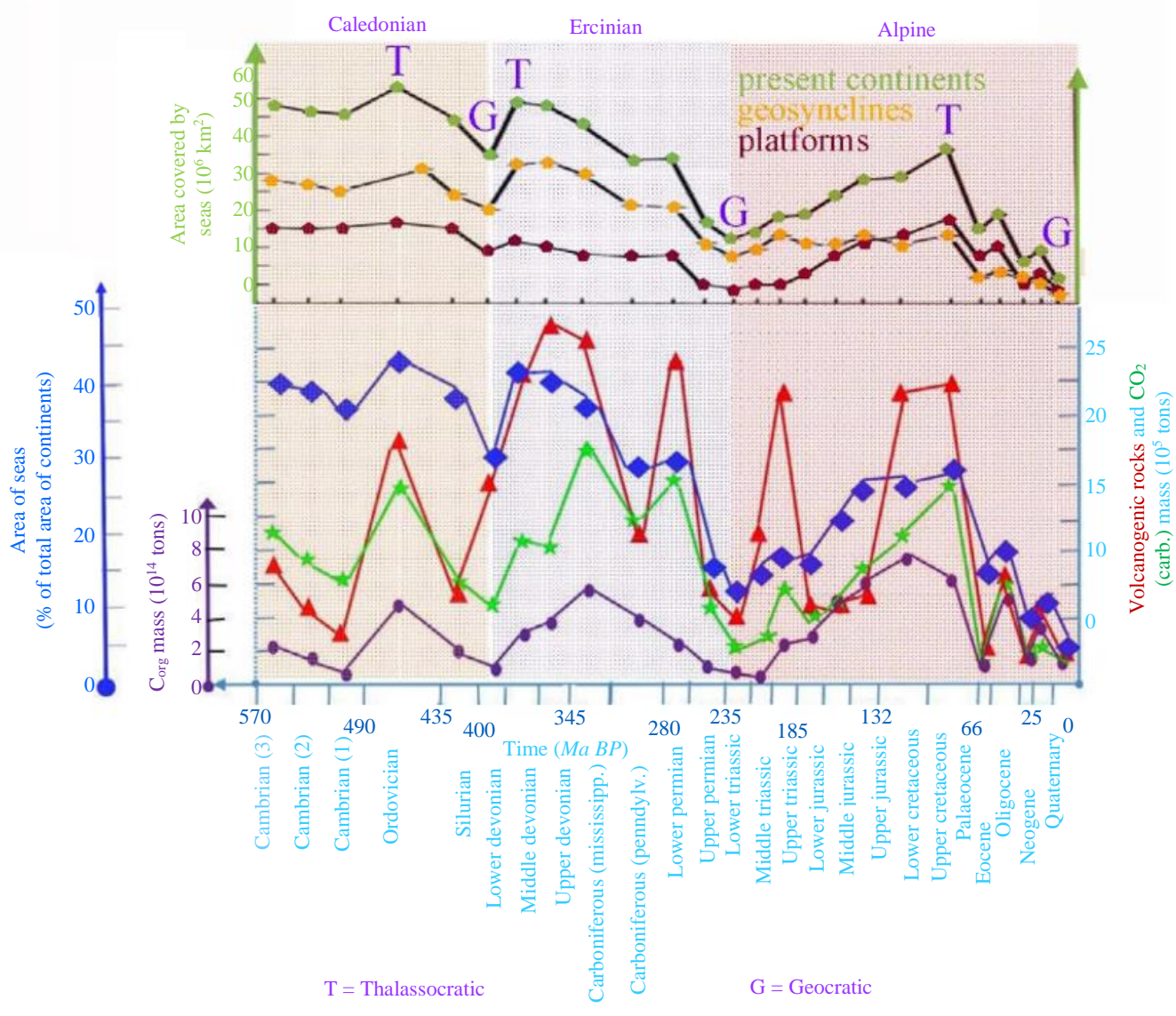

Fig. 11: Synthesis of the analysis reported by (Ronov, 1982; Budyko et al., 1987) and here compiled on the basis of a few figures shown by Ronov and co-workers. In the lower plot, the trends are closely similar of (i) volcanogenic rocks (red), (ii) carbonatic rocks (green) and (iii) organic carbon (brown). Thalassocratic and geocratic mean an environment dominated by prevailing seas or continents, respectively. According to the WMT rationale, the alternation of a varying amount of land vs. ocean (upper plot and blue line in the lower plot) depended either (a) on overthrust of lithospheric slabs and consequent varying elevation of platforms and orogens, or (b) on a (presently unknown) variation of the total amount of water stored, respectively, in the atmosphere, in ice sheets, or underground (down to the serpentosphere). Ronov (1982; Budyko et al., 1987) claim that volcanism and soil exhalation of carbon compounds is the needed basic supply for the preservation and survival of life. Unpublished figure 


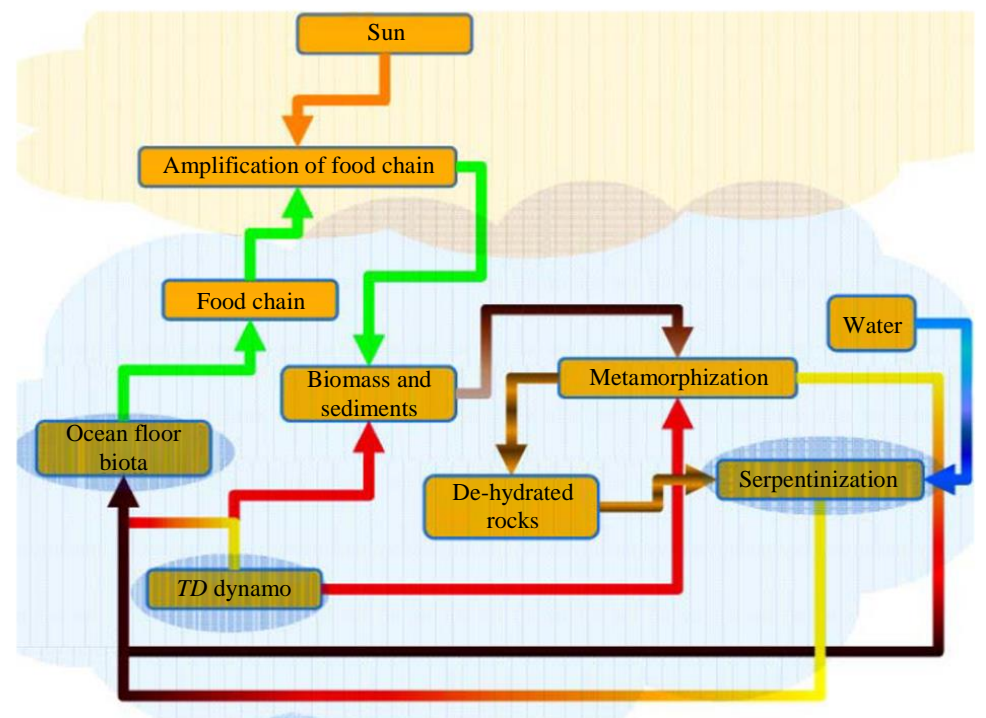

Fig. 12: Carbon cycle of the Earth and of other planetary objects. The three leading drivers of climate are here clearly evidenced: (i) TD dynamo, (ii) the ocean floor biota and (iii) serpentinization. Soil exhalation of $\mathrm{CH}_{4}$, of geogas and of geothermal flow is supplied either by the TD dynamo and/or by serpentinization and it contributes to the development of the biosphere and also to metamorphization, including dehydration of sediments and of rocks that were hydrated by serpentinization. The lowest part of the flow-diagram also applies to every planetary object, where, in addition to the TD dynamo and serpentinization, a leading driver can be operative, that is identified with the biosphere, independent of the more or less faint solar irradiance. When solar irradiance is sufficiently strong, like in the case of the Earth, the Sun contributes as an additional very efficient energy source. Solar energy is thus captured and amplified by the biosphere through higher-levels of Darwinian evolution and through differentiation of species and life forms. Therefore, the upper part of the flow-diagram applies only to a limited fraction of planetary objects. In general, life can be realistically supposed to exist even on a planetary object that is very far from the Sun, where solar irradiance is negligible, even though all life-forms must remain strictly underneath the surface of the planetary object, eventually inside an ocean of water. Unpublished figure

\section{Impacts and the Exploitation of the Sun-Earth Electrical Circuit}

\section{Impacts-Solid Earth}

The Earth is a unique system. However, just for clarity and description purpose, a distinction is to be made between the impact of climate change on three subsystems, i.e., on solid Earth, on ocean/atmosphere and on the biosphere, even though the overall relations are always to be considered according to three engines.

In addition, consider the generally unnoticed wrong way to conceive the biosphere, that is supposed to be an independent and optional additional component passively added to the system. In contrast, the biosphere is the leading component of the Earth system, while the physical and chemical drivers are just complementary facets (e.g., consider that the overwhelming largest part of the Earth' crust derives from metamorphization of biomass). On the other hand, the present level of understanding of natural science is such that the role of the biosphere can be tackled

\footnotetext{
${ }^{32}$ Plate tectonics and global isostasy are rebutted by observational evidence. The Earth expansion hypothesis has several controversial and unsupported features. "Surge tectonics" has no support for the speculated flow of matter perpendicularly to Earth's surface. Wrench tectonics is a bottom-up model
}

by relying on some biological understanding that is comparatively much smaller than for physical and chemical phenomena.

Moreover, a warning is needed for the reader who is not an Earth scientist. Every geodynamic model unavoidably relies on a large amount of speculation, i.e., on a bottom-up exploratory approach, with no top-down confirmatory analysis. The scheme that is here presented is one possible "simple" and top-down scenario consistent with several observations-but, all observations cannot be listed in a few pages. This model does not exclude that other schemes can be eventually proposed ${ }^{32}$. Every disagreement must be discriminated by means of crucial observations. In this respect, the perspective of the "Stellar Transformer", which is outlined in the section on Actions, is just a way to search for truly unprecedented observational evidence, aimed to focus on the role of e.m. phenomena, in addition to thermal and gravitational drivers. As far as the present available evidence is concerned, the scheme that is

that speculates about continental-size drifts. Etc. If one eventually finds support for a given model, he can propose a top-down interpretation. But, if no additional information is available, the choice must favor the top-down model that requires the least amount of speculation. 
here proposed is aimed to look for a self-consistent "simple" and top-down explanation by means of the hopefully least amount of speculation.

A simplified scenario of the impact on solid Earth can be outlined by means of uplift of superswells along the MORs network (Fig. 6). The uplift plays a central role. The mechanism of WMT (see section on The Propagation up to Earth's Surface and to the Atmosphere) leads to the formation of continents and mountains (by thrusting and/or folding), that are later destroyed by weathering and erosion with a creation/destruction cycle of $\sim 100-200$ Ma (likely $\sim 180 \mathrm{Ma}$ ). In addition, Africa seems to be the continent that is comparatively more strongly rooted on the mantle. E.g., the mean thickness of the lithosphere seems to be generally thicker in Africa than in other continents (see section on The Propagation up to Earth's Surface and to the Atmosphere and concerning geodynamics see also below).

North America regularly drifts westward, while Alaska is experiencing a rapid and huge northward trip, sliding on the slope of the huge superswell with center located, roughly, around the Easter Island. Eurasia is pushed westward by the loading tide of the Pacific Ocean (see section on The Propagation up to Earth's Surface and to the Atmosphere).

Consider the Morocco/Japan megashear line. The eastern termination of Eurasia leaves in the rear some area that is no more loaded and "plugged" by continental lithosphere. Hence, a phenomenon occurs that can easily explain the formation of the so-called island arcs, composed of volcanic islands that, upon a detailed analysis, are regularly spaced, from one another. The present easternmost signature, in the Pacific Ocean, of such a westward drift is the so-called "Andesite Line".

The western termination of the Morocco/Japan megashear is located slightly south of the Gibraltar Straights and north of the Atlas Mountains. The first macro-signature at Earth's surface is associated to the counterclockwise rotation of the Iberian Peninsula that determined the opening of the Gulf of Biscay. Then, a slab of lithosphere detached from the Iberian Peninsula and moved eastward-relative to Eurasia-leaving in the rear the Balearic Islands, then Sardinia and later also Corsica at the time when the slab collided with the main body of Eurasia. The collision occurred slightly north of the city of La Spezia (Cinque Terre). The Italian Peninsula is presently rotating counterclockwise and undulating (Fig. 13). Sicily is the northward extension of Africa. The "hinge" between Sicily (Africa) and the Italian Peninsula

${ }^{33}$ Friction heat is the prime driver both for Etna and for island arcs volcanism, although the island arc morphology-i.e., an almost circular pattern with regular spacing of islands-can be exactly explained by specific tectonic setting and dynamics. In contrast, Etna is a point-like mechanical "hinge" that produces friction heat, with no other analogy with island arcs. Advection simply means is close to the Messina Straights. The huge friction-heat of the "hinge" is the primary supply for Etna. Etna accumulates energy (because sea water is insufficient for thermal advection) and once every $\sim 2-3$ years it erupts thus ensuring the energy balance ${ }^{33}$.

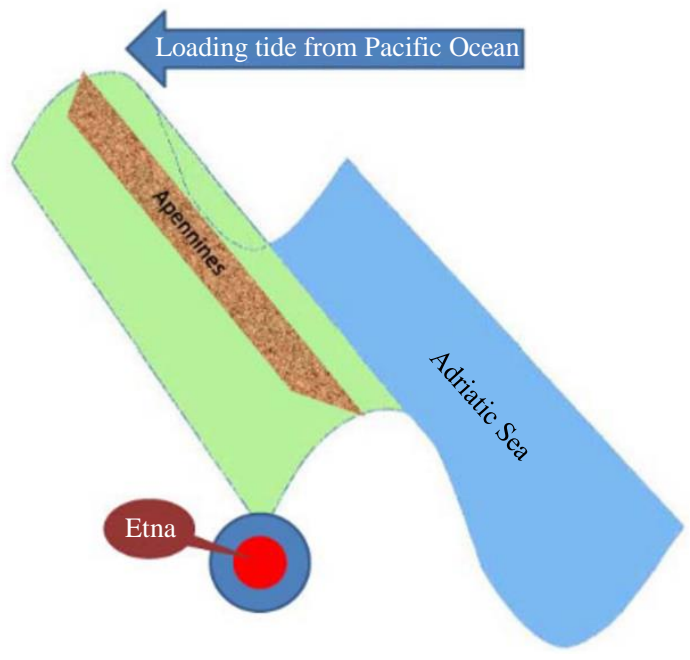

Fig. 13: Origin of seismicity in the Italian peninsula. Eurasia is pushed westward by the Pacific loading tide. Sicily is a promontory of Africa and opposes the motion at a "hinge" close to the Messina Straights. Etna is the security valve to release the friction heat of the "hinge". See text. After (Gregori et al., 2017). With kind permission of New Concepts in Global Tectonics

The counterclockwise rotation of the Italian Peninsula leaves, in the rear, the Tyrrhenian Sea, that is characterized by diffuse submerged volcanism. The largest volcano of the Mediterranean region is the Marsili Mountain, i.e., a huge submerged volcanic complex located at the center of the Calabria Arc. In addition, unlike the eastern side, the western side of the Apennines is characterized by geothermal flux and volcanism.

Italy looks therefore like a slab compressed against the Alps. A reasonably regular spacing occurs of areasalong the Italian Peninsula-that are characterized by clusters of larger and smaller stress and strain (Fig. 14). The rotation with respect to the Alps occurs along a set of right-faults ${ }^{34}$ crossing through the whole Po Valley and south of the Alps. The two points of major strainor some kind of secondary "hinges"-correspond to the two most important hot springs (SPA) in the Po Valleyi.e., Acqui Terme and Abano Terme that is located in

transport of heat by motion of hot matter. The less viscous fluids move first. When they are insufficient, the local temperature increases, until eventually producing melt and magma, which is a newly available fluid, etc.

${ }^{34} \mathrm{~A}$ right (left) fault is such that an observer located on one side of the fault observes the other side to move right (left). 
the area of the Colli Euganei and Colli Berici, the mysterious and unique, presently extinct, volcanic complex in the Po Valley (Fig. 14).

Consider the superswell that is roughly centered at the middle of the Indian Ocean. It can be called "Kerguelen superswell". India is violently sliding NNE-ward and is responsible for the ongoing huge uplift of Himalaya and also for a left-faulting relative to Eurasia mainland .... The Sunda Archipelago and the Moluccas Sea, are regions of violent tectonic contrast-between the India push and the island arc formation mechanism of the western Pacific boundary. Hence, the Sunda Archipelago, the Moluccas Sea and the surrounding areas are characterized by intense seismicity and volcanism. Volcanoes are supplied either by sea-urchin spikes, or by friction-heat.

The northward sliding of Arabia originates the Zagros Mountains and the Persian Gulf, while the Red Sea progressively opens and the Anatolian Peninsula experiences a very rapid and steady counterclockwise rotation-documented by GPS records-while it penetrates like a cleft, into the Aegean Sea.

Therefore, the Aegean Sea is more seismic than the Balkan Peninsula, that is more seismic than the Italian Peninsula. The same phenomenon is also the likely responsible for the uplift of the Caucasus and, maybe, also for the formation of the Carpathians.

The heat released in the Banda Sea is the likely origin for the trigger of El Niño, but the Banda Sea geothermal anomaly also originates a cyclic uplift of Indochina and the consequent crustal stress that propagates along two branches. One branch runs along the China coast until north of Beijing, until a "hinge" point close to a great fault responsible for huge earthquakes. The other branch runs south of Himalaya, until a "hinge" close to Karakoram and such a push is probably responsible for the steady uplift of this anomalous extended orogen.

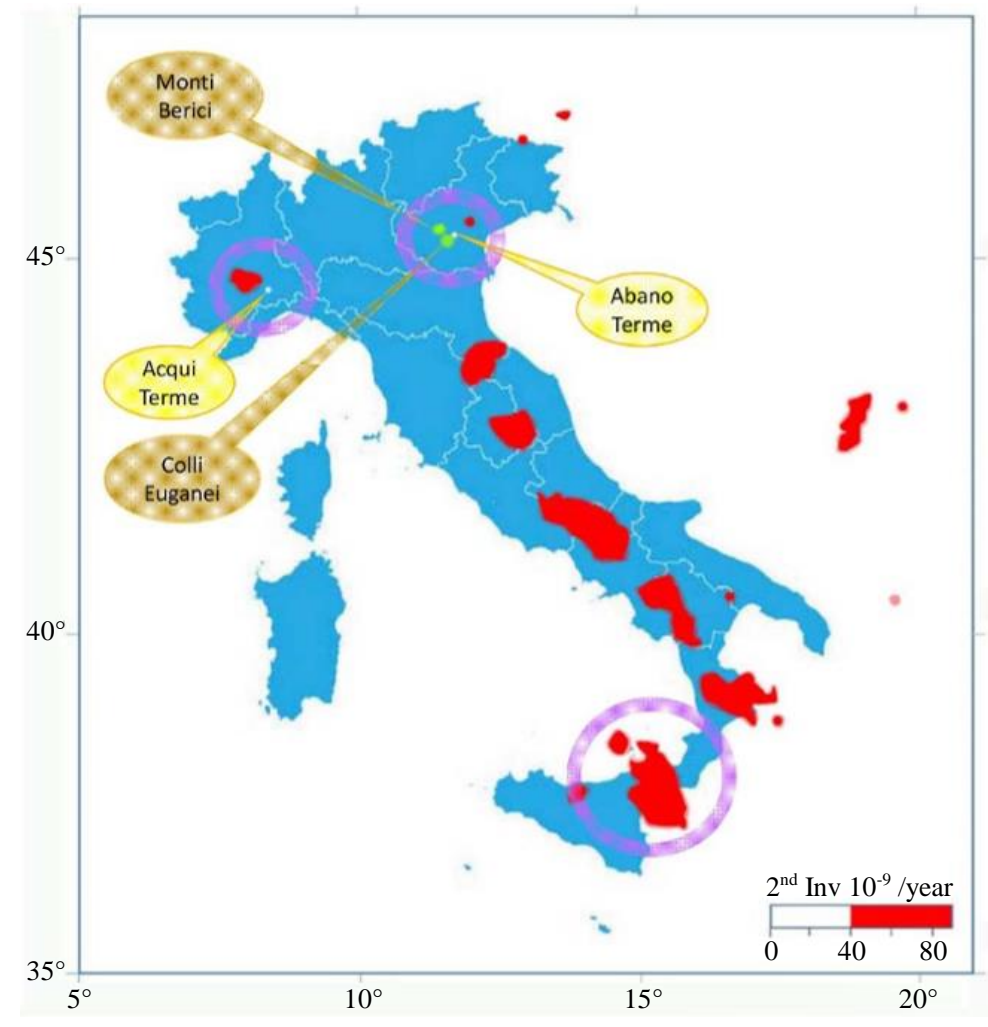

Fig. 14: Approximate sketch, showing the indicative location of relative total strain rate maxima (in red) and the location of Acqui Terme and of Abano Terme (white points) and of Colli Euganei and Monti Berici (light-green patches). For precise locations refer to original maps. Three guessed "hinges" are indicated by light-violet circles. The main "hinge" is close to the Messina Straights and is the likely supply for the present Etna's activity, being however complicated (maybe) by the partial overlapping with the Aeolian Islands volcanism. Two secondary "hinges" are responsible for the present intense hot springs that supply the two unique SPA resources of the Po valley. The Colli Euganei and Monti Berici are the unique anomalous volcanic phenomena found in the Po Valley, that were erupted during the middle Eocene (50-40 Ma ago) and during the lower Oligocene ( 33-30 Ma ago). They are the possible consequence of a strong "paleo-hinge" that, maybe, was located in this area. This interpretation is consistent with the WMT geodynamic model. The total-strain-rate data source is Riguzzi et al., (2012). See text. Unpublished figure 
Concerning Himalaya, the uplift is in progress since 40 Ma ago and determined the formation of the so-called Third Pole, due to the large extension of the ice-cover that severely affects the planetary atmospheric circulation (see section on Impacts-Ocean and Atmosphere).

Also note that the detachment of Madagascar from Africa is associated to a local great endogenous source that, owing to the "plugging" by the thick South African craton ${ }^{35}$, ought to be responsible also for the formation of diamond-bearing kimberlites (see section on The Propagation up to Earth's Surface and to the Atmosphere).

The top-down understanding of this whole scenario is therefore closely related to the capability to envisage where and why a comparatively larger amount of endogenous heat is released-either at present or in the past.

A substantial step forward can be achieved by consideration of the interaction between different seaurchin spikes. In fact, it can be shown that, owing to energy stability and to Hamilton's, different spikes must drift and coalesce into a unique spike, etc.

There are locations, however, where a spike is in equilibrium, as the spike can move and coalesce into either one direction or another. It can be shown that this occurs when a spike is located either at a vertex or along an edge of an exact tetrahedron (Fig. 15)-where, however, every edge ought to be eventually curved in order to fit the shape of Earth's surface (in fact, a spike penetrates as long as it finds the solid body of the Earth $)^{36}$. It has to be emphasized that the tetrahedron pattern is a rigorous and compulsory requirement of Hamilton's, much like the law by which a current must expand in space as much as possible. No speculation is implied by the tetrahedron.

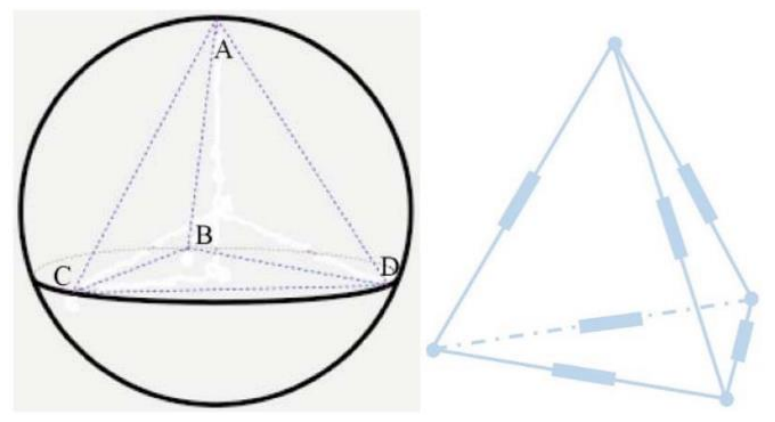

Fig. 15: [Left] A tetrahedron inscribed in a sphere. [Right] A system of deep conductors in the Earth. Every conductor ought to be suitably curved to match Earth's curvature. See text. Unpublished figure

\footnotetext{
${ }^{35} \mathrm{~A}$ craton is a particularly thick layer of lithosphere and crust.
}

In addition, the spikes must occur aligned along an edge, as they cannot drift and merge, moving along the edge, with either one vertex or the other. Furthermore, owing to dynamic stability, one vertex of the tetrahedron must be located at the spin axis of the Earth. At present, a vertex is located right at the North Pole. The other three vertices define the opposite side of the tetrahedron and are located at the same latitude, i.e., exactly at $24^{\circ} .332 \mathrm{~S}$. This is called the Antarctic face of the tetrahedron. Note that $24^{\circ} .332 \mathrm{~S}$ is almost exactly the latitude of the Tropic of Capricorn $\left(23^{\circ} .43663 \mathrm{~S}\right)$. The spatial difference, along a meridian, is $<100 \mathrm{~km}$. Such a tetrahedron symmetry justifies the often noticed and mysterious well-known peculiarity by which a continental and an oceanic area are generally antipodal to each other.

The longitudes of the three vertices of the Antarctic face are located at a relative exact interval of $120^{\circ}$. The better longitudinal location of one vertex seems to be, maybe, just at the very center of Australia (i.e., the Uluru-Kata Tjuta formation). The corresponding deformed (see below) signature at Earth's surface is represented by the nearby location of the Australian Antarctic Discordance (AAD), which is a geological anomaly observed on the ocean floor slightly south of Australia. Also, the latitudinal displacement has a precise reason, as shown below.

Beneath every vertex-and beneath every edge of the tetrahedron-an intense release of heat flow is in progress (Janáčková et al., 1989; Urban and Janackova, 1990; Gregori, 1993). This whole feature can be clearly shown to correspond to the present pattern of the top-axes of the superswells (see below), although with the addition of the role of a few larger-scale superswells. Australia hangs just in the balance over one vertex.

One must take into account the fact that, according to WMT, the lithosphere rapidly slides on the slopes of superswells, due to the ALB lubrication that is particularly effective during a heartbeat (lower plot of Fig. 9). Hence, the signature at Earth's surface of the exact tetrahedron pattern is deformed by the WMT sliding of the lithosphere. Therefore, at Earth's surface the signature loses much of the former regularity-in contrast, the exact pattern was just a strict requirement for the energy stability due to Hamilton's. In this respect, the Circum-Antarctic Ridge is just the deformed signature, at Earth's surface, of the exact deep tetrahedron. Note, in any case, the substantial asymmetry between the Northern Hemisphere-which is characterized by a vertex of the tetrahedron implying a huge release of endogenous energy-and the Southern Hemisphere-that is associated to the edges of the Antarctic face of the tetrahedron.

But the tetrahedron argument must be somewhat changed, due to a crucial additional physical argument of

\footnotetext{
${ }^{36}$ Note that this equilibrium tetrahedron-argument, in terms of an analogy when dealing with gravitation, reminds about the relationship of the Lagrangian points of the Solar System.
} 
"quasi-equilibrium". In fact, one must consider that other sites of equilibrium are to be considered, that are located, respectively, at the center of every face of the tetrahedron. In fact, the generation of sea-urchin spikes is steadily renewing vs. time. Consider, therefore, the spikes that are continuously generated on one face. They rapidly converge and coalesce either into the closest spike, or eventually at the face's center, where they are at equilibrium. The process, however, takes some nonvanishing time and the timescale of the humans is such that the timing can be monitored while the subsystem evolves towards final equilibrium-almost like a frame of a movie by a high-speed camera.

The humans detect the system during the transient evolution towards equilibrium. This is the meaning of the concept of "quasi-equilibrium".

The evidence envisages that, compared the other 3 faces, the Antarctic face experienced a smaller generation of spikes. Hence, the electric currents along the edges of the other 3 faces increased. Thus, it can be shown that the other 3 faces had to expand, thus shrinking the Antarctic face. The final result was that the vertices of the pre-existing tetrahedron were pushed southward.

After some long argument (not here given), it is found that the original tetrahedron thus assumes the shape of an "Orthogonal Quasi-Regular" solid (OQR), while the newly born spikes originated a new tetrahedron, with vertex always at the North Pole. The final pattern is shown in Figs. 16 and 17.

The process can be illustrated in terms of an equilateral triangular pattern EFG that is developed inside the Antarctic face identified with BCD. Note the relative location of the two triads BCD and EFG. The EFG pattern is generated when the former Antarctic face is pushed southward to the exact latitude $51^{\circ} .0576$ S. Each triads show points, which are observed from the bottom face (Fig. 17g), that are aligned along a circle, EFG are Points of the Inner Circle (PIC) and $\mathrm{BCD}$ are Points of the Outer Circle (POC). For future reference in section on The MOR Network, the Deep Tetrahedron/OQR Pattern and Sun-Earth Relations, the proposed interpretation is that a tetrahedron was first formed, that-owing to "quasi equilibrium"'-pushed the Antarctic face southward until the later EFG location, while, at the same time, a newly born tetrahedron pattern BCD was generated. Therefore, the pattern of Fig. $17 \mathrm{~g}$ refers to a comparatively older signature represented by EFG, while a more recent feature is represented by $\mathrm{BCD}$. These inferences derive from a rigorous application of Hamilton's according to the "quasi equilibrium" rationale.

All these features can be recognized by a careful analysis of the morphology at Earth's surface (Fig. 18).

According to the most likely interpretation, an older tetrahedron pattern ought to be represented by the
North Pole vertex plus by the three PIC points. The largest fraction of the present MOR network is representative of this pattern, that was later WMT deformed due to the "quasi-equilibrium" evolution that generated a new tetrahedron pattern with the same North Pole vertex and with base at the POC points $\mathrm{BCD}$. The best signature of this new pattern seems to be the Carlsberg Ridge. Table 2 shows the association between the exact deep conductors and Earth's surface signatures that were slightly deformed by WMT. In particular, the former central Australia signature shifted at the latitude of the AAD.

The most violent WMT deformation is represented by the clockwise rotation of South America, that is still in progress (Fig. 19). The process is likely to be originated by a strong relative maximum of endogenous heat release known as South American Anomaly (SAA), that is roughly located somewhere around and south of Brazil.

The Circum-Antarctic Ridge was interrupted. Thus, the Scotia arc was generated-according to a mechanism that can be shown to be analogous to the Pacific Ocean island arcs-including the formation of the Antarctic Peninsula. The morphology of volcanism of the Transantarctic Mountains (de Vries et al., 2018) is similar to hotspot volcanism, rather than with island arc volcanism envisaging an origin typical of MOR volcanoes, rather than of Andean volcanoes. This pattern also explains why the Andean coast is much more seismic than the North American Pacific coast and also, e.g., why the gravity anomalies in South America are much more pronounced than in North America (Fig. 20).

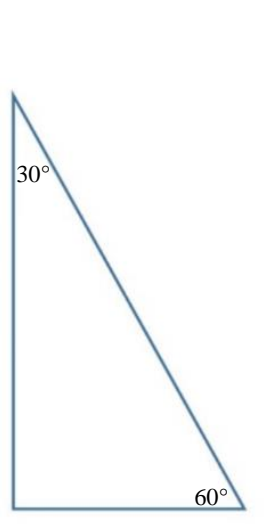

(a)

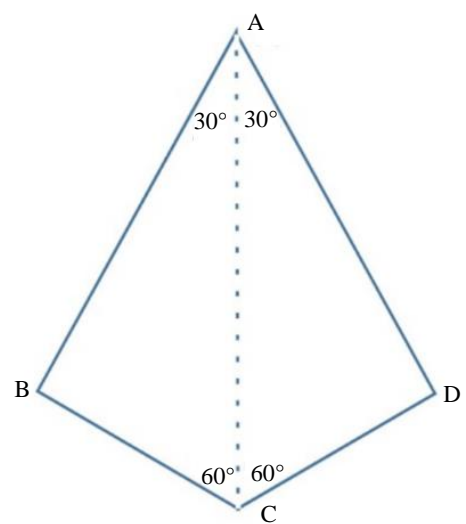

(b)
Fig. 16: Definition of an "orthogonal kite", according to Chester (2014), who defined the construction of the OQR, although only by means of "artistic" arguments. (a) Consider a rectangular triangle equal to half an equilateral triangle. (b) Consider two triangles as in (a) and join them along their hypotenuse. This is a "orthogonal kite" used to construct a peculiar solid, i.e., the OQR defined in Fig. 17. Unpublished figure 

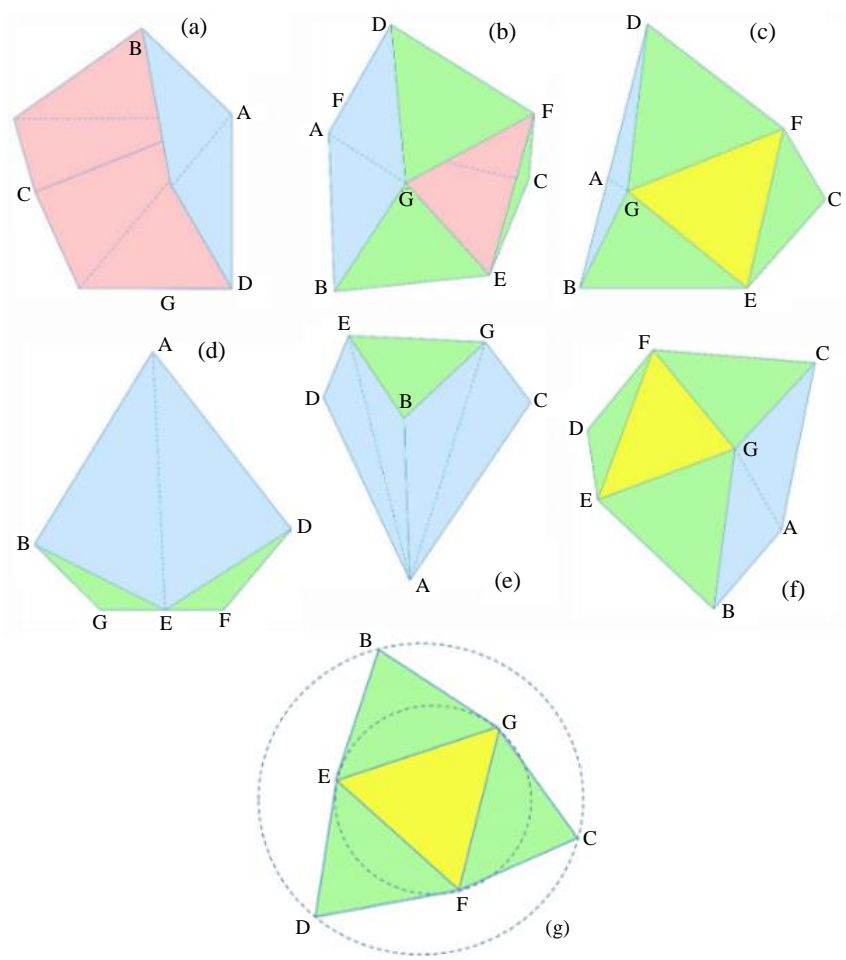

Fig. 17: Construct of an OQR starting from three "orthogonal kites" defined in Fig. 16. (a) Join three "orthogonal kites" at their apex, thus reminding about an elongated tetrahedron. The external surface of the solid is colored in blue and the interior is colored in red. (b) Put three quasi-equilateral triangles (colored in green) in order to close, partially, the empty space underneath the resulting surface shown in (a). A hole is left open, which is shown in red, as it is the interior of the surface shown in (a). (c) Close this hole by an equilateral triangle (yellow). (d)-(f) Thus, the solid OQR is defined. (g) The OQR is observed from beneath the yellow base, with the purpose to distinguish the Points of the Outer Circle (or POC points), i.e., B, C, D and the Points of the Inner Circle (or PIC points), i.e., E, F, G. The whole cartoon here shown is only indicative and descriptive, eventually out of scale. Unpublished figure

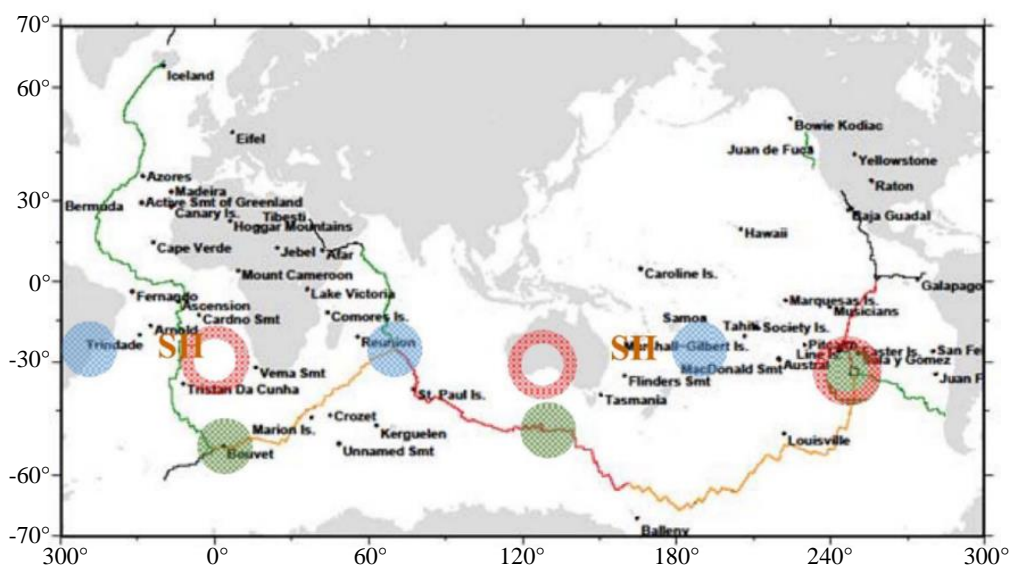

Fig. 18: Global MOR system and hotspot distribution, where different ridges are shown by different colors. The blue circles show the three POC points. The PIC points are shown by red (open) circles. The green circles show, respectively, the Bouvet Triple Junction, the Australian/Antarctic Discordance (AAD) and the Easter Island Triple Junction. Note that the "SH" class of isotopic chemism of basalt (indicated by red capital letters), which is the lowest level in the 5-level energy-hierarchy of ocean basalt (description in preparation), is found in two comparably small regions. One is located in the area around Tubuai, Rurutu, etc. and the other is at its approximately antipodal area in the Atlantic Ocean, around St. Helena Island, at roughly the same latitude and at the longitude of the Bouvet Triple Junction. The figure is modified with specific additions after Masalu (2015). Since the journal Earth Sciences is "Open Access" online, permission for reproduction is kindly granted 

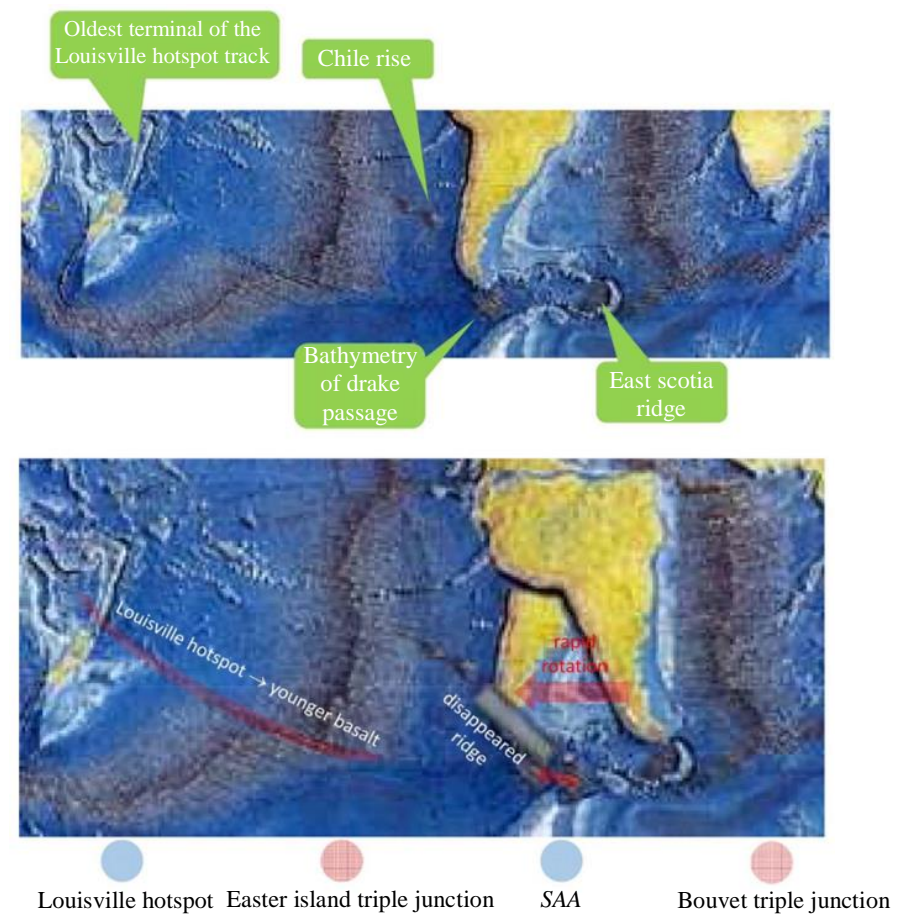

Fig. 19: [Upper image] A detail of the southern ocean belt, extracted from the map of the ocean floor by (Heezen and Tharp, 1977). A few names were added to show a few remarkable features. Source at http://www.marietharp.com. Reproduced by free permission. Retrieved in (Anonymous, 2008). [Lower image] A proposed geodynamic pattern for the generation of the Scotia arc. The background topography and bathymetry is the same as the upper image. The track of the Louisville hotspot is known to span approximately the same time interval of the Hawai'i hotspot, i.e., $\sim 70 \mathrm{Ma}$. It is speculated that a clockwise rotation of South America caused the disappearance of a segment of the former MOR. Owing to the volcanic characteristics, the Transantarctic Mountains look to be the remnants of the signature of a former MOR segment. The lowest small red arrow denotes a possible westward drift of the anomalous bathymetry of the Drake Passage, which is shown in the upper image. The blue and red circles drawn at the map-bottom are the same as in Fig. 18. See text. The background image of South America shows the presumed orientation before rotation, although the location of the barycenter of South America ought to be displaced eastward, upon considering the sliding on the slope of the MAR superswell. Unpublished figure

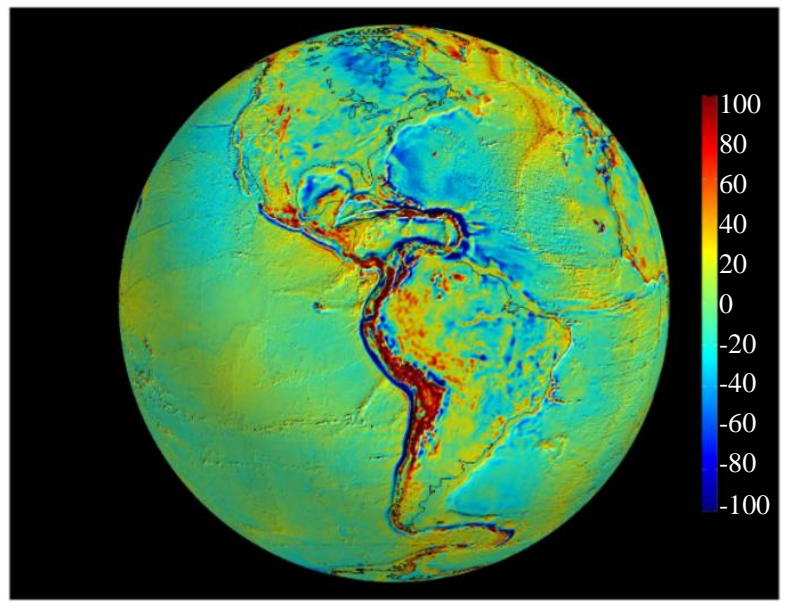

Fig. 20: "The GRACE Intermediate Field 48 (GIF48 from UT-CSR) field model is an improved mean gravity field that combines GRACE observations and terrestrial gravity information The terrestrial gravity information was taken from the DTU10 global gravity field. Shown here are the so-called free air gravity deviations from an ideal ellipsoidal Earth model, in units of mgal. Areas colored yellow, orange, or red are areas where the actual gravity field is larger than the featureless-Earth model predicts, while the progressively darker shades of blue indicate places where the gravity field is less. [Data source: ftp://ftp.csr.utexas.edu/pub/grace/GIF48/GIF48.TXT]" Figure and captions after (Anonymous, 2018). NASA copyright free policy 
Table 2: Guessed location of the tetrahedron vertices and peculiarities located at the center of every face of the tetrahedron Guessed location of the tetrahedron vertices

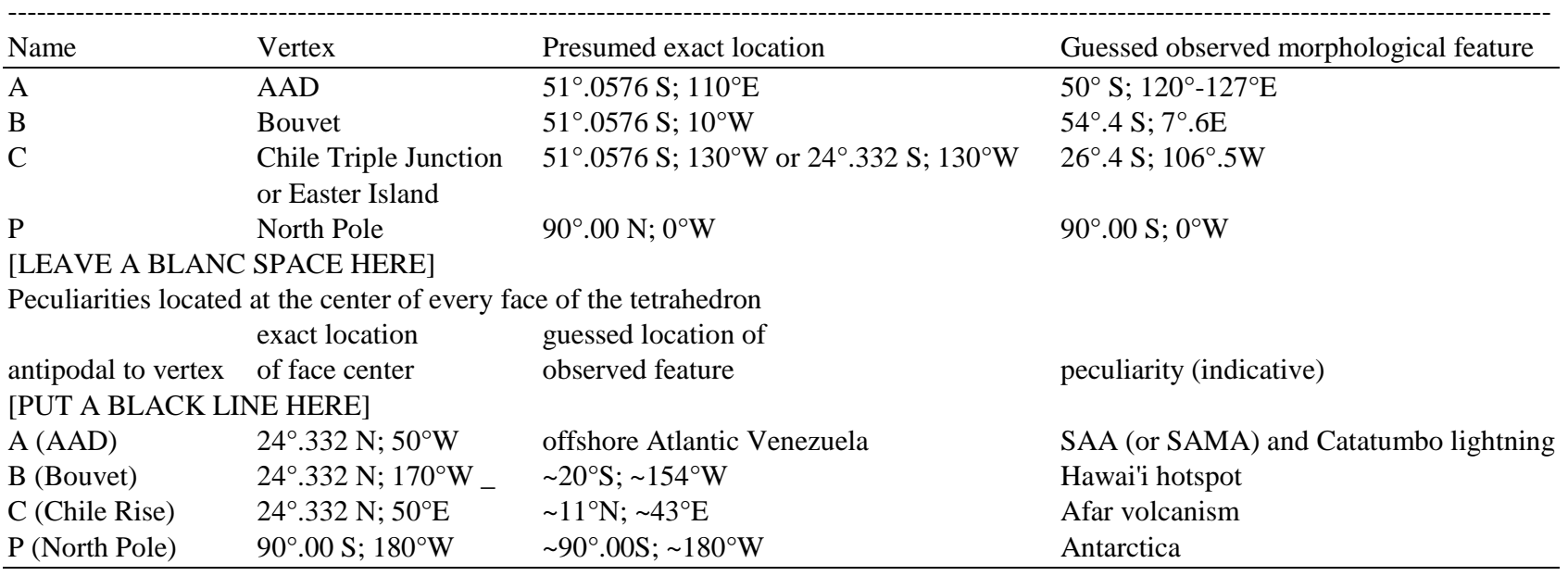

Therefore, the tetrahedron/OQR argument is a topdown explanation (which is, at present, the unique proposed interpretation) for the otherwise unexplained regular pattern of the MORs network.

The tetrahedron/OQR shows the locus of more intense release of endogenous heat, that is the main cause of climate change. The focus shifts therefore on the impact on the ocean/atmosphere system of such a heat release. However, there is a need to understand how these morphological features can impact climate.

\section{The MOR Network, the Deep Tetrahedron/OQR Pattern and Sun-Earth Relations}

The three edges of the Antarctic face-i.e., of the face of the tetrahedron/OQR that is opposed to the North Poleare identified with the Circum-Antarctic Ridge.

The three edges with a terminal at the North Pole are identified: (i) With the Mid-Atlantic Ridge (MAR), from the Bouvet Triple Junction through the North Pole; (ii) with the line, in the eastern Pacific Ocean, from the Chile Triple Junction, through the Easter Island, through the East Pacific Rise (EPR) and California, then through the New Madrid seismic area, through Dallas and Winnipeg (gravity anomaly), through the Hudson Bay, through the North Pole; (iii) with the line from the central Australia edge through the Baikal rift through the North Pole.

Consider the deep exact tetrahedron/OQR pattern-i.e., forget about the deformed signature at Earth's surface that is only the scar of a deep process and according to the timing mentioned in section on Impacts-Solid Earth.

\footnotetext{
${ }^{37} \mathrm{An}$ additional confirmation-of the role of IMF on the orientation of the spin axis of a planetary object-is derived from the mysterious 4 minisatellites of the binary system Pluto/Charon, including the very curious orientation of their respective 4 spin axis (they are solid rocky bodies $10-40 \mathrm{~km}$ in diameter that spin very fast). Their orbital plane is identical for all 4 minisatellites and the plane is perpendicular to the
}

Thus, e.m. induction must be considered by the solar wind into such a peculiar system of electric conductors.

A precise computation is impossible, as several physical data are missing (electrical conductivity $\sigma$ of the components, short-circuiting by the surrounding environment, depth and cross-section of the conductors, role of sea-urchin spikes like antennae with respect to e.m. induction by the solar wind, etc.). It is possible, however, to tackle the problem in terms of a few successive approximations.

A quantitative, though approximate, estimate, that cannot be here explained in detail, deals with a simple and exact tetrahedron circuit (Fig. 15). The latitude of the vertices of the Antarctic face is exactly $24^{\circ} .332 \mathrm{~S}$. If a computation is carried out by assuming that e.m. induction is felt only by a given face that directly "links" the flux of the impinging IMF-while other faces that are in the "shadow" have no e.m. induction, being screened by the "shadowing" face-it is found that the optimum energy condition is attained when the tilt of the rotation axis of the Earth is exactly $24^{\circ} .332$, which is almost the same as the latitude of the Tropic of Capricorn. If the OQR deformation is considered, the approximate correction can be computed by some algebra (not here explained) and the conclusion is that the tilt of the spin axis of the Earth ought to be just $24^{\circ}$ minus a few degrees.

The slight discrepancy with the Tropic of Capricorn can derive either (more likely) from the approximations introduced in the computation, or from the fact that the Earth spin-axis is still reorienting itself until it will attain the exact value of $24^{\circ} .332^{37}$.

ecliptic plane. Also the 4 spin axes denote a close reciprocal coupling. In fact-unlike what is generally stated-the solar wind has a non-neutral charge (see section on Actions). Hence, when a fast-spinning body is charged, it generates a magnetic moment and the 4 minisatellites are thus correctly magnetically coupled one another. Both planes, either of the Pluto/Charon system or of the 4 minisatellites, are perpendicular to the 


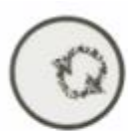

(a)

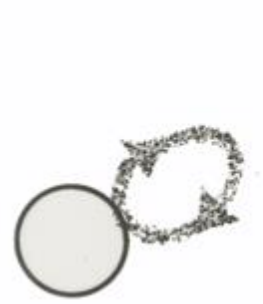

(c)

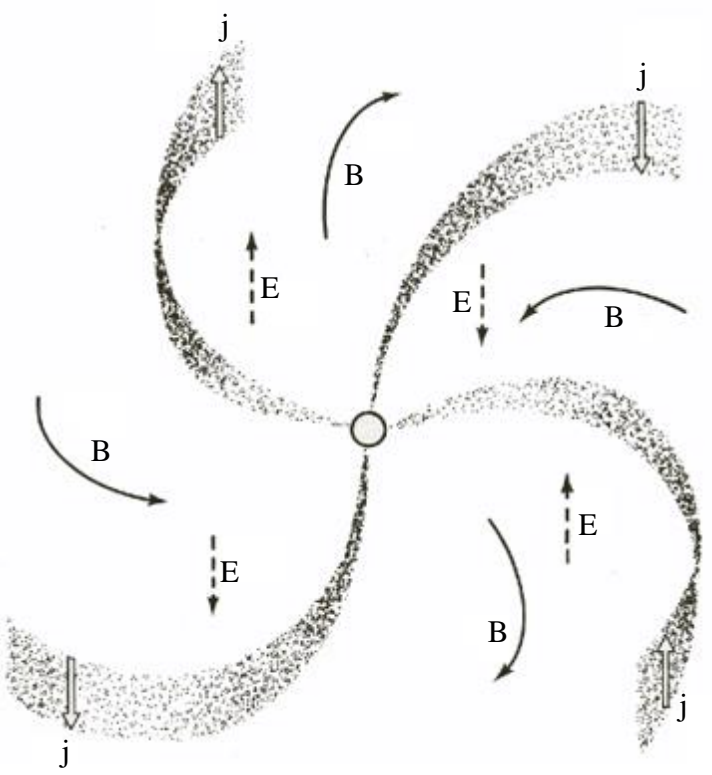

Fig. 21: [upper figure] A Coronal Mass Ejection (CME) projects through space $\mathbf{j}$-loops that characterize the well-known classical observed Parker spiral pattern of interplanetary environment shown in the lower plot, that is caused by the rotation of the Sun. Owing to the almost infinite conductivity of the solar wind and to the frozen-in flux approximation, the $\mathbf{B}_{\text {int }}$ pattern is associated with a corresponding pattern of the interplanetary electric field $\mathbf{E}_{\text {int, }}$ while, owing to the Maxwell's relation curl $\mathbf{B}=4 \pi \mathbf{j}$, some kind of huge "blades" of currents $\mathbf{j}$ called "Heliospheric neutral Sheet" (HCS) must separate the different interplanetary sectors. Unpublished figure

ecliptic plane, i.e., consistently with an electrically charged solar wind At present, these curious and precise observed features can be explained in no other way, thus being a direct proof of the non-neutral electric charge of the solar wind.

${ }^{38}$ Since the Sun is mostly plasma, the poles and equator have different rotation speeds, i.e., solar polar rotation is $\sim 36$ days and the Earth
This conclusion applies to the whole planet Earth, but a concern is about the instant e.m. induction inside the deep conductor underground, i.e., either in the exact tetrahedron, or inside its exact $\mathrm{OQR}$ variation. Therefore, the detail must be assessed of the inducing e.m. signal and the distinction is needed between the interplanetary signal and the signal detected inside every given edge of the deep conductor. In fact, the magnetosphere acts almost like a "modem" that determines the e.m. field at every point inside and around the Earth.

In the interplanetary environment, the kinetic energy density of the solar wind overwhelms the magnetic energy density. Hence, $\mathbf{B}$ is transported by the expanding solar corona (B is "frozen-in" the solar wind, according to Alfvén's theorem). But B field lines cannot break, while the Sun spins (at $\sim 27$ day period), hence the Parker spiral is formed (Fig. 21). The most usual observed configuration is composed of 4 sectors of $\mathbf{B}$ polarity direction (away $\rightarrow$ forward $\rightarrow$ away $\rightarrow$ forward), except when the Sun is "quiet", i.e., when all sunspots coalesce into a unique larger sunspot and only 2 sectors occur (away $\rightarrow$ forward). Note that sectors, in general, are not equal one another. Since curl $\mathbf{B}=4 \pi \mathbf{j}$, some thin $\mathbf{j}$-sheets separate one sector from the other. This whole sheet is called "Heliospheric Current Sheet" (HCS). Since the solar equatorial rotation period is $\sim 27$ days, the Earth ${ }^{38}$ experiences this rotation every $\sim 30$ days, due to the differential relative orbital motions, crossing either 4 or 2 times the HCS.

The magnetosphere is characterized by an intricate $\mathbf{j}$ pattern (Fig. 22). Close to the Earth, the $\mathbf{B}$ energy density is larger than the kinetic energy density. Hence, particles are trapped by $\mathbf{B}$ and the radiation belts are formed. In the transition region-where $\mathbf{B}$ and kinetic energy density are comparable each other-an intricate and time-varying $\mathbf{j}$ system is formed.

A system of closed $\mathbf{j}$-loops (Fig. 22) runs along the tail and the tail looks like two huge solenoids. As observed from the Earth, for an away sector, the northern solenoid looks with a counterclockwise $\mathbf{j}$ and the southern solenoid with a clockwise $\mathbf{j}$. During a HCS crossing, every $\mathbf{j}$ is reversed. The transition process looks like a thin "knife" that cuts through the magnetosphere and progressively changes the $\mathbf{j}$ system. The process occurs during the time needed by the solar wind (at a typical mean speed of $\sim 400 \mathrm{~km} \mathrm{sec}^{-}$ $\left.{ }^{1}\right)$ to cross until the end of the tail of the

experiences this at $\sim 40$ days due to differential relative orbital motions of Earth and Sun. In other words, the Earth moves relative to the Sun's rotation, thus the Earth has moved forward by the time the Sun makes a complete revolution. 
magnetosphere ${ }^{39}$ (i.e., $\sim 1000 \mathrm{R}_{\mathrm{E}}$ ). This time lag is of the order of a few hours ${ }^{40}$.

Consider the effects produced on the Earth by a crossing of the HCS. A first effect ought to occur on the variation of the length of the day (1.o.d.) and on pole motion. In fact, consider the instant orientation of the (dipolar) magnetic moment of the Earth ${ }^{41}$ that is subject to the action by magnetospheric current system of Fig. 22.

The crossing of the HCS exchanges the comparative role of the two solenoids. Whenever a perfect north/south symmetry occurs of the magnetosphere, the final effect is therefore null. But, at every other instant of time with no perfect symmetry, an effect is expected to occur, due to the structural asymmetry of the two lobes and eventually depending on the intensity of the currents inside the HCS, that is casually related to the intensity of the IMF.

This phenomenon is to be investigated, as the magnitude of the effect cannot be foreseen. A concern to be clarified is whether and when the effect is eventually stronger depending on the inclination of the magnetic dipole axis with respect to the impinging IMF.
The e.m. induction in the deep conductor of the Earth is related to the electric field $\mathbf{E}$ inside the magnetosphere (Fig. 23). But, in this respect, a distinction must be made depending on the mechanism that generates the temporarily observed $\mathbf{E}$ and different mechanisms are operative at different times.

One mechanism is originated in the solar wind (hence it is denoted by the acronym SW). In fact, B field lines are characterized by an extremely high conductivity (due to spiraling electrons around a field line), hence a $\mathbf{B}$ field line is approximately equipotential. The $\mathbf{E}$ that exists in the interplanetary environment is directly projected along $\mathbf{B}$ field-lines over the polar caps. The SW mechanism generates the $\mathbf{E}$ pattern of Fig. 23 (first and second image). Upon considering $\mathbf{B}$ records at some prechosen conventional suitable set of observatories, located inside one polar cap (and simultaneously inside both polar caps), it is possible to infer a circular $\mathbf{j}$-pattern that flows either clockwise or counterclockwise depending on the interplanetary sector polarity (Svalgaard vortex).
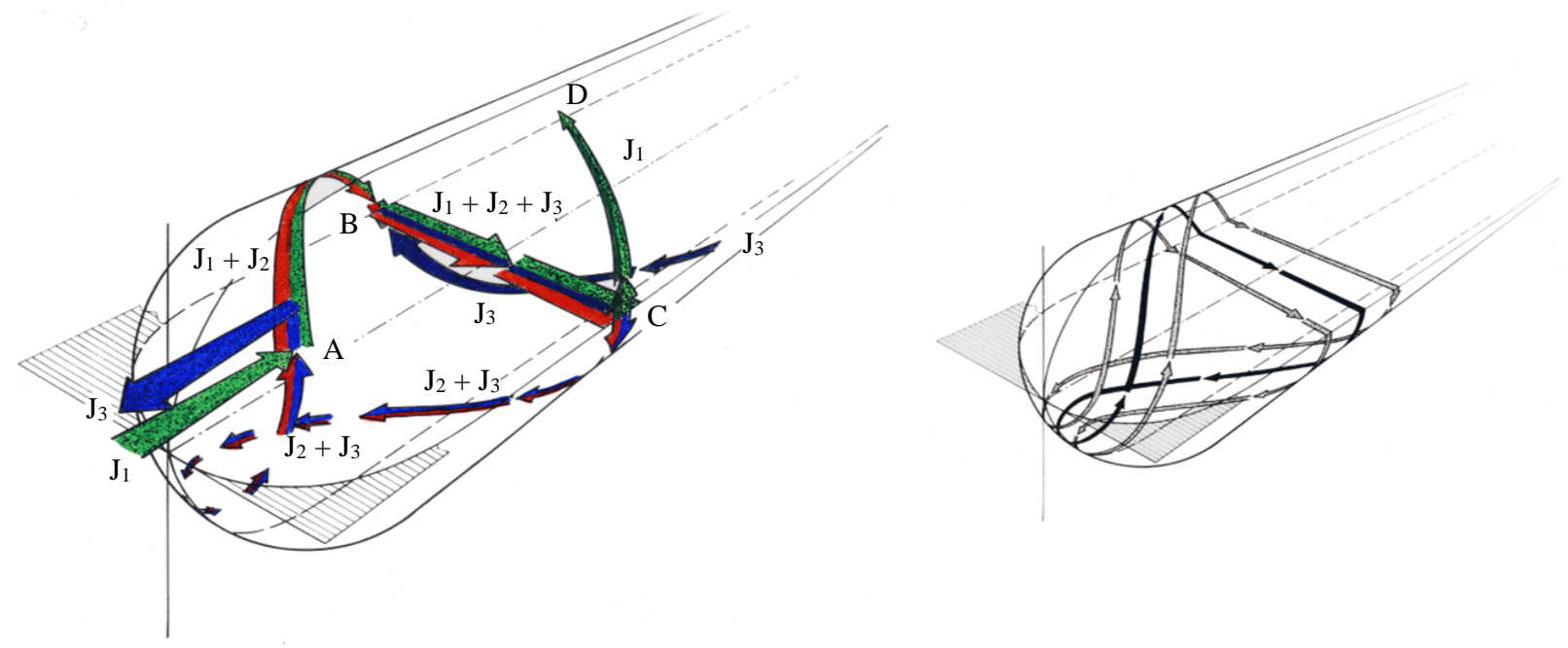

Fig. 22: [left figure] The cartoon shows three topological patterns of $\mathbf{j}$-loops in the magnetosphere (case history of an away $\mathbf{B}_{\text {int }}$ interplanetary sector, every arrow refers to a current $\mathbf{j}$ being due either to protons or to electrons). One $\mathbf{j}$-loop, here denoted by $\mathbf{J}_{1}$, envelops the northern lobe of the magnetospheric tail, looking from the Sun like a counterclockwise solenoid with a j flowing downward. Another similar clockwise solenoid, here denoted by $\mathrm{J}_{3}$, envelops the southern lobe, with a $\mathbf{j}$ flowing sunward. The third $\mathbf{j}$-loop, here denoted by $\mathbf{J}_{2}$, is here represented in a substantially simplified form, as its pattern is better shown in the right figure. [right figure] The $\mathbf{j}$-pattern denoted as $J_{2}$ in the left figure is composed of closed loops. The present cartoon shows two (not three) $J_{2}$ loops of this kind. The simplest possible $\mathbf{j}$-loop is shown as a solid black line. One (only and only one) additional $\mathbf{j}$-loop, in grey, is drawn being a representative of a large set composed of an infinite number of $\mathbf{j}$-loops, all having the same geometrical pattern, although of different extension downstream. Check that only one of these (grey) $\mathbf{j}$-loops is represented in this figure and thus realize that a conjugation exists between different site vs. tail distance. These $\mathrm{J}_{2}$ loops represent some new kind of trapping orbits, although it appears very unlikely either (i) to afford to measure this phenomenon, or (ii) even to presume that this ideal condition can remain with no severe disturbance that destroys it, that is originated by the fluctuations of the solar wind flow. See text. After Gregori (1998; 1999; 2001). With kind permission of the late Wilfried Schröder

\footnotetext{
${ }^{39} \mathrm{R}_{\mathrm{E}}$ is an Earth's radius.

${ }^{40}$ The confirmation is given by the so-called magnetospheric substorms, that occur when a gap ("plasma cavity") occurs in the solar wind. In fact, a substorm typically lasts $\sim 2-3 \mathrm{~h}$.
}

\footnotetext{
${ }^{41}$ The magnetic dipole axis is inclined $\sim 11^{\circ}$ with respect to the Earth's spin axis. Hence, the magnetic moment-much like a spinning top-describes a diurnal cone around the instant orientation of the spin axis.
} 


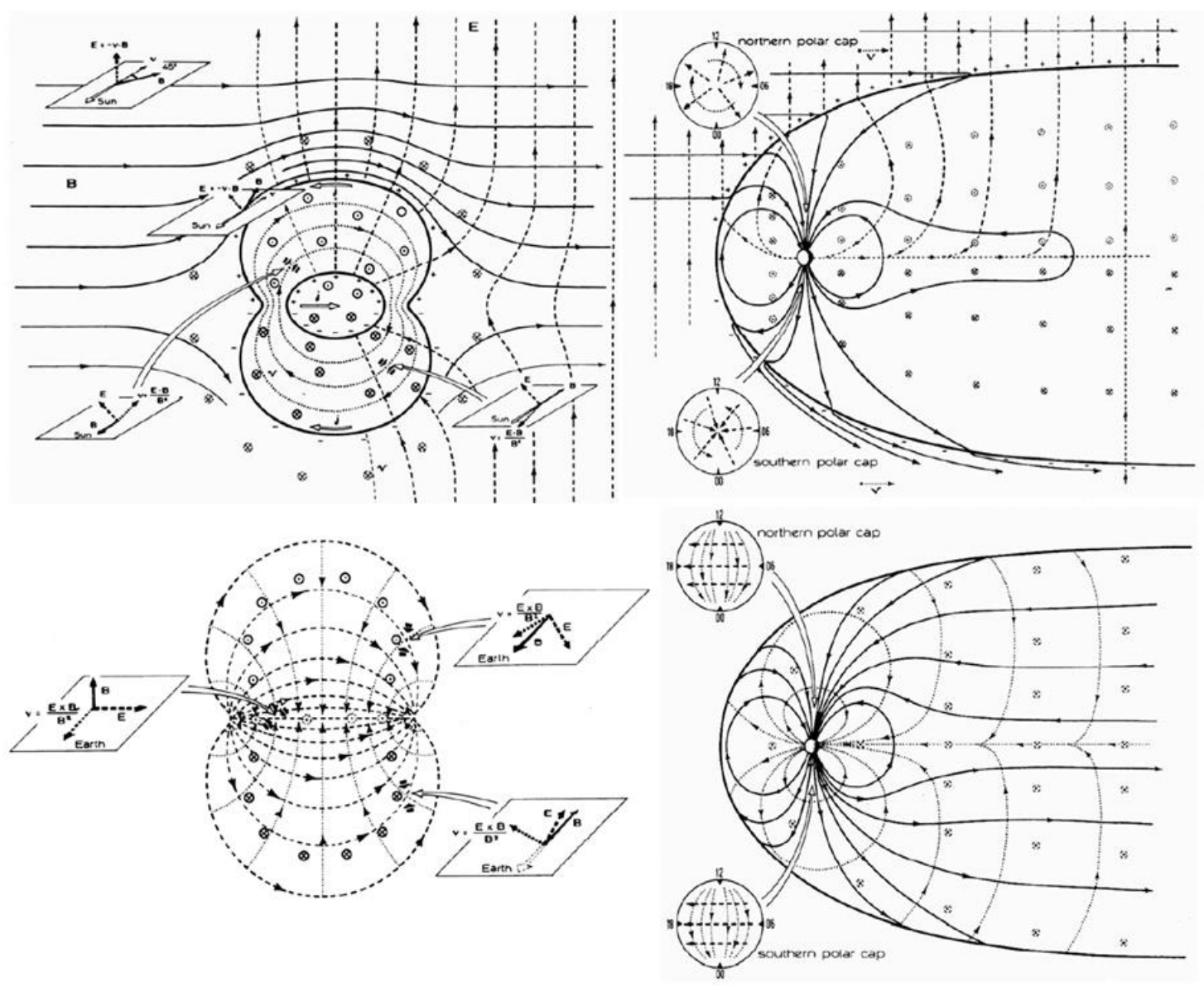

Fig. 23: [First and second image] Electric field $\mathbf{E}$ generation in the magnetosphere by the SW mechanism for an away sector. Crosssection and meridional cross-section, of the tail, as observed from the Earth. [Third and fourth image] The same according to the IO, TP and FI mechanisms. Cross-section and meridional cross-section of the tail. When dealing with a forward sector, the direction of some vectors has to be suitably reversed. See text. After Gregori $(1992 ; 1998 ; 1999)$. With kind permission of SIF

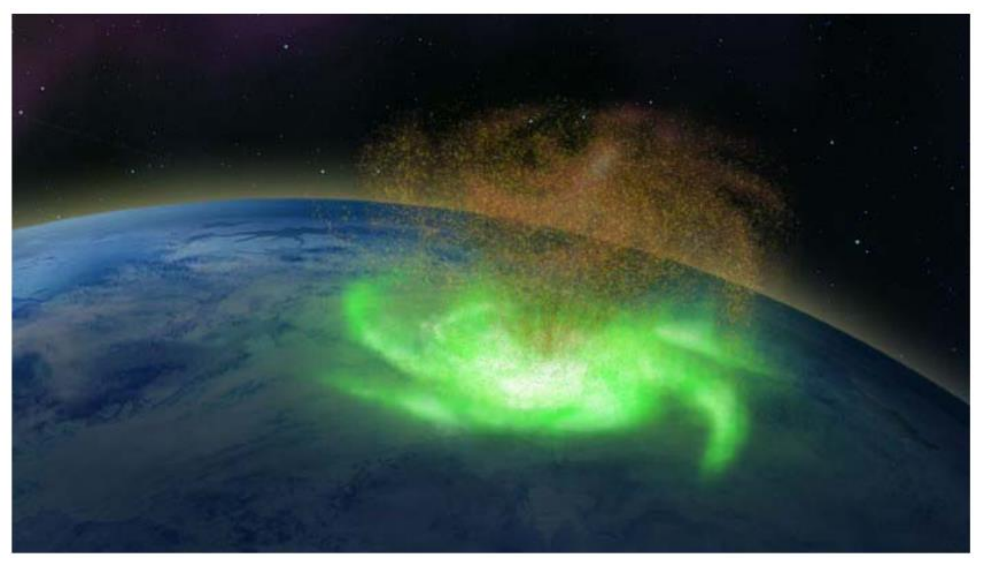

Fig. 24: Cartoon synthesizing the observations carried out by the Chinese satellite DMSP F16 and displaying a "space hurricane". This observational pattern can be interpreted in terms of a huge flow, over the northern polar cap, of Field Aligned Currents (FACs), with a likely pentagonal pattern, consistently with the (Scott, 2015) argument. The clockwise skew rotation is consistent with the circular polar bulk velocity pattern in the second plot of Fig. 23. This interpretation is other than the model proposed by the authors (Zhang et al., 2021), i.e., the pattern that is here shown was inferred with no bias by the interpretation that is here proposed. See text. After Xing (2021). Reproduced with kind permission of Qing-He Zhang 
The typical geometry resembles a circle. Hence, this state of the magnetosphere can be called "O" state.

A recently available direct observation is shown in Fig. 24. A huge particle precipitation was observed by the Chinese satellite DMSP F16. It was a Field Aligned Current (FAC) that must display a polygonal pattern (Scott, $2015)^{42}$. The sector polarity was away (Zhang et al., 2021) ${ }^{43}$. Hence, the bulk $\mathbf{v}$ pattern on the northern polar cap must be clockwise, as per the second plot in Fig. 23. This "space hurricane" looks like a FAC impinging with a pentagonal pattern that converges while spiraling clockwise. The associated (Hall) currents are, as a standard, counterclockwise, consistently with the expectation of the Svalgaard vortex.

Another similar effect ought to be detectable (not yet done) by means of the so-called for Auroral Electrojet (AE) index that is computed by means of the $\mathbf{B}$ records simultaneously measured at a select set of observatories in an auroral zone. The AE index represents therefore the j that flows inside polar auroras. Unlike in the Northern Hemisphere $\mathbf{j}$, in the Southern Hemisphere the observed $\mathbf{j}$ ought to be influenced by the e.m. induction in the deep Circum-Antarctic conductor. Hence, the asymmetry between the two hemispheres ought to be a signature of the deep tetrahedron/OQR conductor.

Other 3 mechanisms can be considered for the generation of $\mathbf{E}$ inside the magnetosphere. All of them generate an E-pattern of identical topology (Fig. 23, third and fourth image). One mechanism is associated to the poor electrical conductivity of the Earth's Ionosphere (IO mechanism). When a $\mathbf{j}$ crosses through the ionosphere, a potential drop is generated along the $\mathbf{j}$-loop. The potential drop is projected back into the magnetosphere along equipotential B field-lines. The other 2 mechanisms are associated to a violent injection of a plasma into the magnetosphere-either from the front side (FI mechanism), or from the tail (TP mechanism for tail plasma) and operate like an MHD generator.

In every one of the IO, FI or TP case histories, a dawndusk (or dusk-dawn) $\mathbf{E}$ crosses through the polar cap. The best evident signature is given by the comparatively seldom occurring polar aurorae (TPA, for trans-polar aurorae) that are observed inside the polar cap aligned with the solarantisolar direction. Several auroral images are available, not here shown, of aurorae on a polar cap and remind about a $\Theta$. Hence, this can be called " $\Theta$ " state of the magnetosphere.

Summarizing, maybe, the available data sets and investigations on the $\mathrm{AE}$ index display some northern/southern asymmetry that, however, must be investigated in separated sets, depending on the " $\mathrm{O}$ " or " $\Theta$ " state of the magnetosphere. In fact, mixing the two case histories into a unique set, is like computing a mean between fishes and birds.

\footnotetext{
${ }^{42}$ Several morphological features on the Earth clearly envisage a likely origin by a huge electrical discharge (Burn, 2015).

${ }^{43}$ It should be pointed out, however, that the model implemented by these authors cannot be here shared, as their model, consistently with the
}

In addition, in general the Circum-Antarctic deep conductor ought to be investigated by considering the north/south asymmetry inside the whole ensemble of geomagnetic observatories that are located inside two suitable symmetrical high-latitude belts. Some lesser observed effect ought to permit to assess whether the leading e.m. induction occurs inside the older exact deep conductor at $\sim 51^{\circ} \mathrm{S}$ (PIC points tetrahedron), or rather in the temporary and newly born OQR pattern at $\sim 23^{\circ}-24^{\circ} \mathrm{S}$.

This statement exhausts the discussion on the CircumAntarctic deep conductor while, concerning the northsouth tetrahedron edges, the $\mathbf{E}$ ought to be known that is roughly parallel to these edges.

With SW mechanism, the $\mathbf{E}$ inside the magnetosphere is perpendicular to the ecliptic plane with orientation that changes while crossing the HCS. In contrast, when the mechanism is either IO, or TP, or FI, the $\mathbf{E}$ inside the magnetosphere is always roughly perpendicular to the ecliptic plane, although it is always from the equator poleward, independent of the away or forward sector polarity.

Therefore, an astute analysis-of the instant records at the geomagnetic observatories that are located reasonably close to either one of the 3 tetrahedron/OQR edges-ought to give a signature of the amount and timing of the instant e.m. induction inside every edge.

Note that the instant geomagnetic records ought to detect a phenomenon that lasts a few hours and that occurs at a mean rate of one event every 1-2 weeks, that ought to be closely correlated also with solar rotation. In addition, the phenomenon ought to be detected only for longitudes related to the expected location of every edge, while the phenomenon should not be detected when referring to other longitudinal sectors.

Summarizing, except the Svagaard vortex and TPAs, the effects inside the tetrahedron/OQR associated to the $\mathbf{E}$ field inside the magnetosphere are-at present-not monitored and not understood.

\section{Impacts-Ocean and Atmosphere and the Exploitation of the Sun-Earth Electrical Circuit}

The dramatic evidence of Fig. 9 clearly suggests huge climate changes occurred during the whole Earth's history, at the pace of heartbeats, defined by a "heartbeat unit", i.e., $1 \mathrm{hbu}=27.4 \mathrm{Ma}$. Note that several conventional boundaries in the standard geological timescale often comprises several hbu.

It is impossible by a few statements to illustrate an item that ought to require an encyclopedia. Just a few mentions can be given and by distinguishing historical and present processes. In either case, the unicity of the Earth system ought to be stressed, composed altogether of solid Earth, ocean and atmosphere.

present fashionable assumption, refers to the vertical IMF component and neglects the overwhelming IMF component in the ecliptic plane-that, in fact, was correctly measured as shown by Figure 1 of (Zhang et al., 2021). 
Violent climate changes have always occurred. One dramatic phenomenon was represented by atmospheric density, that increases with increasing soil exhalation and decreases when the Earth, during a FR, misses the magnetospheric shield and is spoiled off by solar wind interaction. Thus, huge variations occurred in atmospheric density and composition. Today, the large flying creatures of some ancient past would be unable to fly, as they lack the Archimedean force. According to several evidences, the atmosphere was sometimes even some 5-10 times denser than at present (Levenspiel, 2000; Levenspiel et al., 2000).

Also, the chemical composition eventually varied by a comparable amount, depending on the varying soil exhalation. According to present experiments inside greenhouses, several forms of vegetation increase by several times both size and volume, when the atmospheric density of $\mathrm{CO}_{2}$ is artificially largely increased. This result is consistent with the aforementioned Ronov's "principle of preservation of life", as $\mathrm{CO}_{2}$ and $\mathrm{CH}_{4}$ are the primary "fuel" for life development.

Huge amounts of almost ubiquitous $\mathrm{CH}_{4}$ exhalation are observed everywhere (Judd and Hovland, 2007). Occasionally, beachings are reported of deep oceanic fauna, such as giant squids. The phenomenon is reported during several months from different parts of the world. Then, for several years no event is observed. The likely explanation is that an anomalous increase of $\mathrm{CH}_{4}$ exhalation and hydrothermal venting from the ocean floor-simultaneous over the globe-is such that the local biosphere eventually moves at a shallower depth while searching for less polluted water. Then, the large pressure variation eventually causes death by embolism.

This is consistent with clear paleo-oceanic evidence that shows that several of the largest extinctions during Earth's history occurred from excess concentration of $\mathrm{CH}_{4}$ in ocean water, caused by an anomalous heating of $\mathrm{CH}_{4}$ clathrates. Every great extinction event is always reported corresponding to a heartbeat.

The primary driver of extinctions was the frequency of the encounters of the Solar System with interstellar clouds that caused several FRs and subsequent anomalous large production of endogenous energy. Consider, however, that several FRs can occur during the paroxysmal stage of a heartbeat and the effect of every FR is to be integrated and time-delayed by a different amount depending on the extension of the "channel" (see section on The Propagation up to Earth's Surface and to the Atmosphere) for energy transfer to Earth's surface. These items have been accurately taken into account (Gregori, 2002).

In addition, interstellar clouds also contain larger objects that refill the Solar System with meteoroids and comets (Oort reservoir). Hence, also the frequency of large impacts-

${ }^{44}$ Refer also to the independent, authoritative and objective, bipartisan previous study by Quinn (2010). although with larger error-bars-matches with the pace of the Hawai'i-Emperor Seamount proxies of Fig. 9.

Atmospheric $\mathrm{CO}_{2}, \mathrm{CH}_{4}$ and $\mathrm{H}_{2} \mathrm{O}$ are well known to be major greenhouse gases that control atmospheric temperature. The atmospheric concentration of $\mathrm{CO}_{2}$ was monitored during one year by the NASA satellite OCO-2, including seasonal variation (Mearns, 2015a; 2015b), that, however, showed that the contribution originated by anthropic action is irrelevant, or maybe even negligible (Gregori et al., 2018; Gregori, 2020) ${ }^{44}$. The concern is about the comparative greenhouse role of $\mathrm{CH}_{4}$, that, at equal atmospheric concentration, is 23 times more efficient than $\mathrm{CO}_{2}$ (Pappas, 2016). However, no mapping is available for $\mathrm{CH}_{4}$ comparable to $\mathrm{CO}_{2}$ and $\mathrm{CH}_{4}$ is a major component of geogas and is eventually stored inside $\mathrm{CH}_{4}$ clathrates. Its release caused extinctions.

As far as $\mathrm{H}_{2} \mathrm{O}$ is concerned, the focus is on the water cycle. It is impossible to estimate the total amount of water that is stored in different natural reservoirs, i.e., inside (i) the atmosphere, (ii) seas and oceans, (iii) icesheets and also (iv) underground-where water, perhaps, has a comparatively larger concentration in the crust (i.e., above the Moho) and is also a relevant component in the serpentosphere, where it is in the form of supercritical water. Hence, it is impossible to estimate-even approximately-the time variation of the total water content in every aforementioned reservoir.

Neither can the hysteria associated with sea-level variations be indicative, as a sea-level datum refers to one given site and several other concurrent possible effects (tectonic, geothermal, etc.) are to be considered that can explain every observed evidence. Similarly, glacier and icesheet phenomena are very intricate and every occurrence can be explained in several different ways. Hence, sea-level and glacier extension are unreliable proxies, of difficult explanation. Therefore, every data series requires a specific devoted study. These basic difficulties are confirmed from mass monitoring by satellite Gravimetric Records (GRACE and GRACE-FO), as stressed by (Frederikse et al., 2020), getting rid of the hydrologic ("steric") temperature effect, but not of the tectonic disturbance.

Concerning ongoing phenomena, a key role is played by the global electric circuit (symbolically synthesized in Fig. 25). No separation exists between solar phenomena and deep Earth circuits. Solar-terrestrial relations occur either through the "external way" or through the "internal way" (Fig. 26). In general, reference is made to the external way, hence the concern about the so-called spaceweather. The external way is expected to prevail at comparatively higher frequencies. But, at low frequencies, the internal way is crucial, as the impact on the TD dynamo causes huge variations of endogenous energy production with strong climatic impacts. 


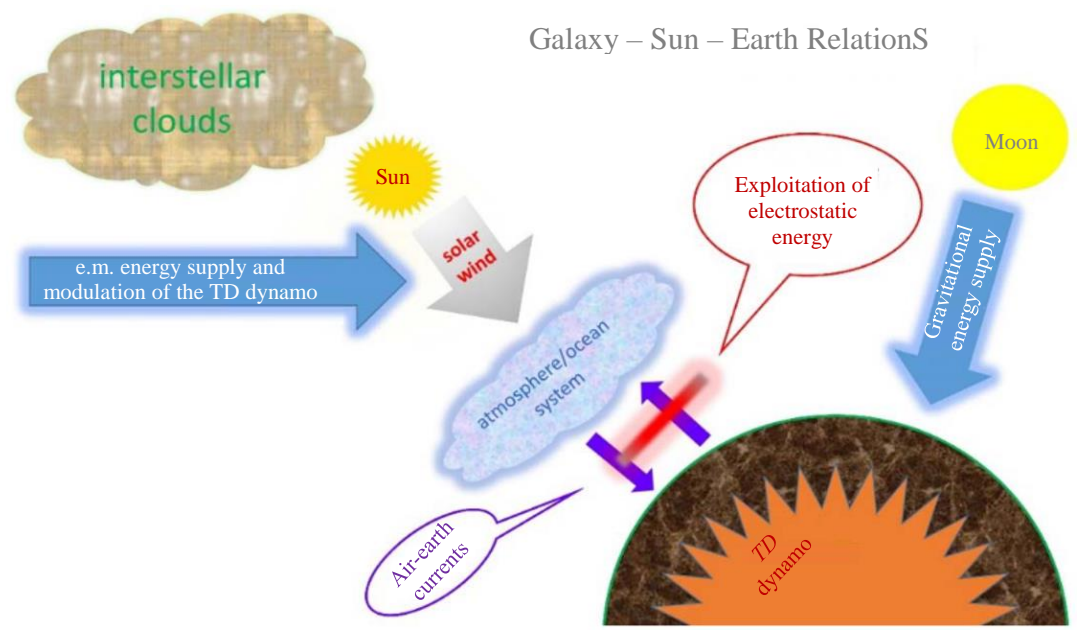

Fig. 25: The global electrical circuit in Galaxy-Sun-Earth relations. See text. After Gregori (2020). With kind permission of Acoustics

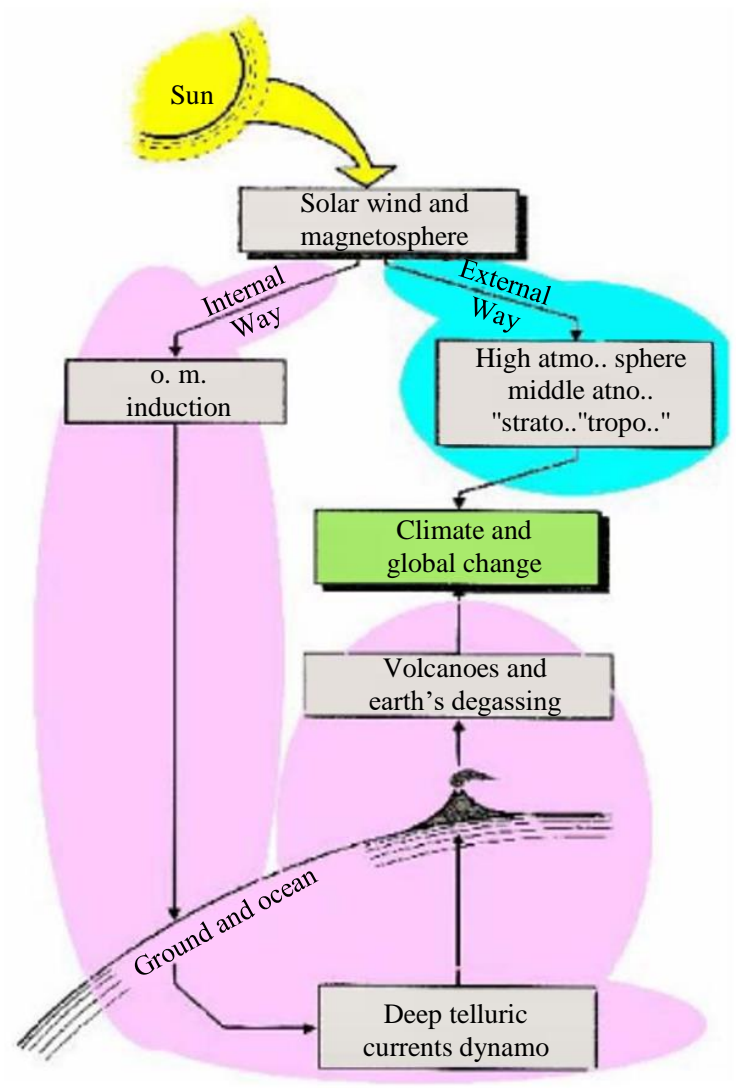

Fig. 26: The "external" and "internal" way in solar-terrestrial relations. After Gregori (1997; 2020). With kind permission of the late Wilfried Schröder

A crucial role is played by the Cowling dynamo that transforms the kinetic energy of a convection cell into e.m. energy, whenever the medium is more or less partially ionized (Gregori, 2020). That is, thermodynamic highentropy phenomena are transformed into an organized system through e.m. fields. Some most evident manifestations are through the "transient luminous events" (that have often been identified with UFO).

The atmospheric electrical circuit is an unexploited facility that is an almost unlimited reservoir of electrostatic energy, that is directly supplied by the lunar and solar tide, through the TD dynamo. Its practical exploitation is discussed by (Gregori, 2020) and in section on Actions.

With reference to present section about the climate concern, a brief reminder is deserved for a few items.

Himalaya, with its ice cover is just a Third Pole of the Earth and the evidence ${ }^{45}$ shows that its uplift, that occurred during the last $40 \mathrm{Ma}$, ought to have been the main responsible for the present atmospheric circulation, with the origin of the Hadley cells that determine the trade winds, etc. (Tang and Dong, 1997; Dong and Tang, 1997).

El Niño is explained by fluid dynamical models that give justice for all teleconnections, although these models are incapable to envisage the trigger and source of phenomena. A guess is that the low-pressure cell in the Banda Sea (south of Indochina), teleconnected to the Easter Island high pressure cell, is the likely location for the energy input that triggers the event.

The global circulation of the atmosphere/ocean system in the Northern Hemisphere (or the global circulation) seems to be controlled, consistently with the aforementioned guessed origin of the very anomalous Baikal Lake, by energy exhalation from the high-pressure cell of the Baikal Lake Rift, teleconnected with the Alaskan low-pressure cell.

${ }^{45}$ This is likely to be one of the most important recent findings in climatology. 
Tropical storms are generated in the Horn of Africa (roughly in the region of the Afar triangle). Then, they move westward until the Gulf of Mexico, whereoccasionally (roughly, say, $\sim 25$ times per year)-are transformed into violent hurricanes by an abrupt almost step-like increase of Sea Surface Temperature (SST). A hurricane is associated to an energy that is well-known to be almost exclusively related to water evaporation ${ }^{46}$, as winds and kinetic energy amount only to a few $0.1 \%$ of the total energy of the hurricane. The energy of a hurricane is comparable to-or even more violent than-the energy of the strongest ever recorded earthquake or volcanic eruption ${ }^{47}$. The phenomenon looks like in the case that some kind of huge volcanic eruption-comparable with or even more violent than all known historical volcanic eruptions-occurs on the sea floor, while heat is released through advection, due to availability of water, from deep ocean floor to water surface, where heat is transferred through SST to the atmosphere.

The hurricane energy is released through devastating precipitations that occur at and after hurricane landing, where the hurricane loses energy, because it is no more supplied by SST.

The reason is not known of the abrupt increase of $\mathrm{SST}^{48}$. One wonders whether it is only a coincidence the presence of the Chicxulub crater - which is the remnant of a huge impact that is believed to be associated to the extinction of dinosaurs. In effect, a substantial debate was concerned with this item and it is presently agreed that the huge impact preceded the dinosaur extinction by $\sim 300.000$ years. Another independent debate is concerned about the origin of the Chicxulub crater. Hence, the whole topic needs harder thinking.

A possible tentative explanation is that the former huge impact caused a "scar" inside the deep solid Earth. Hence, a "column" of great warmed material was formed. Having a larger temperature, i.e., a larger electrical conductivity, this "column" was the site of intense seaurchin spikes, that maintained a preferential "channel" for energy transfer to Earth's surface. This implied huge soil

\footnotetext{
${ }^{46}$ Several papers also emphasize a particularly intense associated lightning activity (e.g., Leybourne and Gregori, 2020). This is correct, as atmospheric precipitations are closely related to atmospheric electricity-according to the Cowling dynamo mechanism (Gregori, 2020) that determines water agglomeration around condensation nuclei. Cowling dynamo is supplied by thermal convection. Hence, a SST temperature increase enhances thermal convection and also Cowling dynamo and atmospheric activity. Similarly, an increased electrical activity triggers a larger precipitation and enhances the impact of the hurricane.

${ }^{47}$ The total energy of a violent hurricane $\sim 1-2: 5 \times 10^{21 \pm 1} \mathrm{~J}$. The total energy released in one volcanic eruption is between $\sim 2 \times 10^{19}$ and $\sim 10^{20}$ J (Santorini, 1400-1500; Laki, 1783; Tambora, 1815; Coseguina, 1835; Katmai, 1912). The total energy released in one earthquake is: 1960 Chile $\sim 2: 5 \times 10^{23} \mathrm{~J} ; 1964$ Alaska $\sim 7: 5 \times 10^{22} \mathrm{~J} ; 2004$ Sumatra earthquake $\sim 4: 0 \times 10^{22} \mathrm{~J}$ (released at Earth's surface $\sim 1.1 \times 10^{17} \mathrm{~J}$ ). Annual total release for earthquakes $\sim 10^{18} \mathrm{~J}$. Annual heat flow release from the Earth
}

exhalation and impact on the atmosphere. A comparatively rapid climate change followed-that, by 300.000 years, finally determined pushed dinosaurs toward full extinction.

Maybe, the same spike pattern still survives. Thus, the whims of the time-varying availability of endogenous energy are manifested at Earth's surface preferentially where such a "scar" favors an easier heat propagation and abrupt heat release, that occasionally supplies a hurricane.

The hurricanes are called typhoons in the Pacific and Indian Oceans. A phenomenon-that in some way reminds about the Gulf of Mexico-occurs in Madagascar where the track of all typhoons seems to end. The Madagascar morphology is characterized by a phenomenon that is seldomly observed in other areas. The phenomenon is called "lavaka", that seems to be generated by a polygonal (pentagonal?) ${ }^{49}$ huge electrical discharges. Lavakas look like a typical landslide on ground-and are clustered envisaging local crowding of several shallow top-points of sea-urchin spikes. This is consistent with the huge release of endogenous energy that occurs in this wide region, associated with several phenomena, the best known of which are, maybe, the anomalous isotopic chemism of basalt ("Dupal anomaly") and the presence of diamond-bearing kimberlites, or the region of permanent higher release of endogenous $\mathrm{CO}_{2}$ detected by OCO-2, etc. (see section on The Propagation up to Earth's Surface and to the Atmosphere).

Summarizing, hurricanes and typhoons are certainly powered by an anomalous SST that ought to be supplied by endogenous heat. Hurricanes die after landing, while the phenomenon is obviously accompanied by a violent atmospheric electrical and precipitation activity. It is suggested that this occurs either by exhausting the energy budget through intense water precipitation (hurricanes), or through violent airearth electrical discharges (Madagascar) ${ }^{50}$.

Finally, note that all atmospheric phenomena are a primary cause of weathering and erosion of soil, being therefore responsible for the comparatively rapid destruction of mountain ranges and of continents,

$\sim 8 \times 1020 \mathrm{~J}$. Total energy of an astrobleme is: Arizona's crater $\sim 1017$ $\mathrm{J}$; Vredefort ring (South Africa) $\sim 6 \times 1021 \mathrm{~J}$; in Australia (130 Ma BP) $1020 \mathrm{~J}$.

${ }^{48}$ The time resolution of SST maps provided by satellite remote sensing are such that the SST increase occurs by a few hours, or less, which is the time needed by satellite orbits to monitor the Gulf of Mexico. But the e.m. induction caused by an impulse could be responsible for the observed event. This ought to be monitored, however, also by the network of geomagnetic observatories of some region of continental size.

${ }^{49}$ The polygonal feature (Scott, 2015) is characteristic of Field Aligned Currents (FACs), or Birkeland currents and is observed in several electrical discharges both on the Earth and on Jupiter.

${ }^{50} \mathrm{~A}$ lightning is a spectacular emission of photons related to a violent discharge. But a discharge can be very intense, although not visible, such as it can occur for huge air-earth currents that certainly exist, mainly in areas of large crustal fracturing. 
according to the $\sim 100-200 \mathrm{Ma}$ cycle (maybe $\sim 180 \mathrm{Ma}$, i.e., Mortari cycle).

\section{Actions}

Several actions can be implemented aimed to prevent and/or mitigate unwanted consequences of natural catastrophes. Several items are extensively considered by Gregori et al. (2018) and, for brevity purpose, are not here summarized.

These items deal with concern with solid material performance (also in Gregori, 2020), including crustal stress (earthquakes and landslides), volcanic edifices, manmade structures (such as buildings, viaducts, dams, every machinery or reservoir, mobile land or marine vehicles and aircrafts or helicopters, embankments, etc.). The basic common principle is monitoring in real time the onset and evolution of stress and the timing of microfracturing, with a long advance-time for alert, suited to permit, if possible, concrete intervention in order to anticipate damage amplification.

The forecast of hurricane track is another item of concern that, however, ought to be managed by operating an array of magnetometer, with records to be analyzed by a still unpublished algorithm (in preparation).

Other hazards refer to wildfires or flood prevention and management and other minor hazards. But a reminder is here deserved about other two additional kinds of actions: Biosphere monitoring and e.m. phenomena in the natural system (clean energy exploitation).

Biosphere monitoring refers to a permanent endemic regeneration of microorganisms on deep ocean floors. Owing to the ever-changing natural environment associated with soil exhalation, an everlasting change is in progress of this specific flora. The result is the wellknown steady variation of microorganisms, viruses and bacilli, that are at the very beginning of the food web. These items are outside the competence of a physicist, being rather the concern of a microbiologist.

A suitable prevention ought to be, as far as possible, to monitor the ongoing processes at the very start of the chain, i.e., by sampling ocean floor by means of unmanned deep ocean vehicles. The time change of microorganism biota ought to occur at the same rate of climate change. But the biological record, perhaps, has a higher sensitivity and time-resolution.

Other items, in general, deal with the role of e.m. phenomena in the natural system. This perspective is briefly called "electric universe" and with the study of electric currents caused by e.m. induction in deep Earth. This is briefly called "Stellar Transformer" (Leybourne, 2015; Leybourne et al., 2017; Leybourne and Gregori, 2020).

Clean energy exploitation is the main focus of the discussion very briefly outlined in the present paper and concrete actions are therefore here described in some detail.
The procedure to intercept electrostatic energy in the atmospheric electrical circuit (as per Fig. 25) are outlined in Gregori (2020). But the concern is about where, when and why a location can be more or less suited to get the maximum cost/benefit performance.

A distinction deals with the source of the e.m. energy, that can be exploited and that can be located either in the troposphere, or in the ionosphere, or in outer space.

The tropospheric origin is related to meteorology. Storms are associated to intense e.m. activity, consequent to the transformation of thermal/kinetic energy into e.m. energy through the Cowling dynamo (Gregori, 2020). Therefore, every location with an ongoing intense storm is likely to have a great amount of electrostatic energy available. The concern is rather about the hazard represented by lightning discharges that can even destroy the plant. There is need to install a safety lightning-rod, much like-when dealing with a riverone must get rid of violent floods by means of floodplains.

The ionospheric source refers to the poor conductivity of the ionosphere. Whenever solar particles (solar wind or higher energy) precipitate on the Earth's atmosphere, they spread all over the globe. The effect, at every given site, is an electrostatic charge in the ionosphere, which is also amplified by tropospheric phenomena through the Cowling dynamo, with a different instant intensity and evolution depending on location. According to the present knowledge, it is impossible to predict where and when the optimum conditions are to be expected. There is need for long-lasting systematic observations in order to monitor the details of the time evolution of ionospheric currents.

The outer space energy source is directly related to solar wind precipitation on the atmosphere. The phenomenon occurs over polar caps, while middle and low latitudes are screened by the radiation belts, that act like an efficient Faraday shield. In particular, the plasmasphere, which is the innermost large fraction of radiation belts, is $100 \%$ filled with trapped particles.

However, some relevant effect can occur, perhaps, on the occasion of the crossing through a HCS that causes a dramatic change of the electric current in the magnetosphere (Figs. 22 and 23). Observations are needed in order to assess, when and where, the effect is eventually maximum.

For instance, it has to be ascertained whether the distance from a deep conductor is crucial of the location of the plant for e.m. exploitation. That is, the distance ought to be crucial from a tetrahedron/OQR edge.

In addition, the latitude can be important. In fact, the E field is induced in the tetrahedron edge at high latitude. But the induced currents flow along the whole edge. Is this sufficient for energy exploitation also at low latitudes?

In addition, it is even possible that a significant amount of e.m. energy can be exploited only on the occasion of HCS crossing. But, even in this case, since $2-4$ crossings 
occur in 4 weeks, a significant amount of energy ought, perhaps, to be available.

All these items can find a reply only by direct campaigns of observations. The aim of the present paper is to give a rationale to highlight such a perspective.

\section{Conclusion}

The basic bias is caused by false information. The misconception begins in the scientific community that presents science in terms of well-assessed, generally agreed, matters-of-fact. This is fully false, as there are many more items that science cannot understand than items that are wellassessed and understood ${ }^{51}$. In fact, ultimate truth is beyond human reach. In contrast, the different schools of thought like to present their achievements as the pinnacle of understanding and want to defend their leadership. Max Planck, in his autobiography published in 1949, wrote that a wrong idea is not rebutted due to scientific arguments, rather only when its supporters die. Conservatism is a true "virus" for science. The "impact factor" of a journal is the most powerful weapon for conservatism.

"Publish or perish" is the famous adage by Einstein who complained about the excessive rush for publishing several short papers devoted to an extremely limited set of specialists, instead of a smaller number of longer papers devoted to a wider scientific audience.

This same false feeling about the "generally agreed truth" is transferred to mass media, who are always hungry for sensationalism and finally also to legislators and magistrates, who believe that a judicial expert can get rid of matters that are not understood even by the most expertized scientists.

But quite a few misconceptions are reported by present "official" science.

One case history deals with the generally accredited statement that earthquakes cannot be predicted. Earthquake (or volcanic, or landslide, or manmadestructure collapse) predictions are feasible with a degree of certainty comparable to meteorological forecast.

For instance, before World War II, no meteorological forecast was possible, as the available records were collected at a sparse number of meteorological observatories managed by a few scientific institutions. Owing to the need for flight assistance for war missions, a global network of land-based observatories was set up, with fixed ships where no islands were available. Some preliminary, rough-though very effective-maps were broadcasted to all aircrafts (one map every $3 \mathrm{~h}$ ) and every end-route officer could plan the flight.

That is, only a suitable network of observations can provide the basis to issue a forecast. This holds for weather and for crustal stress. If no network of recordings station is available, no seismic forecast is possible, but denying a priori such a possibility is just like being guilty of manslaughter.

Other several items are related to the ongoing climate change that is related to the very anomalous period of time that is located just on top of a heartbeat. In this respect, note that a typical maximum endogenous energy release during a heartbeat lasts $\sim 4-5 \mathrm{Ma}$, while humankind history, since early prehistory, lasted, say, 30,000 years. Correctly, there is a concern about anthropic pollution, but one must be careful to focus on the correct target. In terms of analogy, a patient affected by a most serious and eventually lethal disease cannot rely on an antipyretic medicine and hope to solve his concern.

The concern about greenhouse gases is certainly badly posed. The role of $\mathrm{CO}_{2}, \mathrm{CH}_{4}$ and $\mathrm{H}_{2} \mathrm{O}$ is very poorly understood.

As far as $\mathrm{CO}_{2}$ is concerned, the NASA satellite OCO2 records showed that the anthropic contribution is irrelevant, or maybe even negligible (Mearns, 2015a; 2015b; Gregori et al., 2018; Gregori, 2020).

No comparably precise monitoring is available for $\mathrm{CH}_{4}$, that is certainly an intense and ubiquitous release, an essential soil exhalation for life survival, altogether with $\mathrm{CO}_{2}$ and-at equal concentration- $\mathrm{CH}_{4}$ is 23 time more efficient than $\mathrm{CO}_{2}$ (Pappas, 2016).

Concerning $\mathrm{H}_{2} \mathrm{O}$, at present no estimate can be made of the amount of water that is stored in the atmosphere, in the oceans and mostly in subsoil, either inside the upper crust (above the Moho) or inside the serpentosphere. Hence, the concern is nonsensical about sea-level change, or about ice-sheet melt. In fact, both phenomena are controlled by a huge amount of concurrent and often conflicting, drivers and it is impossible to interpret-in any meaningful way-every observational data series, which is eventually per se also dramatic.

We must be quite concerned about climate change and about pollution. Humankind is a time-varying component of the natural system, with a time-evolving space-density impact and energy consumption. "Pollution" is every action by humankind, although every action can be either a damage or a "medicine" to prevent unwanted trends of climate. But wisdom is fundamental, as well as a correct understanding of the drivers of phenomena.

A basic warning deals with the need for multidisciplinary examination. In general, the scientific community wants to define specific realms of competence for different kinds of specialists. Thus, a set of separate disciplines is envisaged, either for diplomatic reasons or in order to avoid the need to become expert in different topics, but this is a most serious handicap. Indeed, several non-understood phenomena can be easily explained only by dropping some conventional-and ultimately unnatural-barrier. Figure 27 shows a set of such barriers, but a few comments for explanation are needed.

\footnotetext{
${ }^{51}$ The Covid-19 pandemics dramatically emphasizes such a bias.
} 


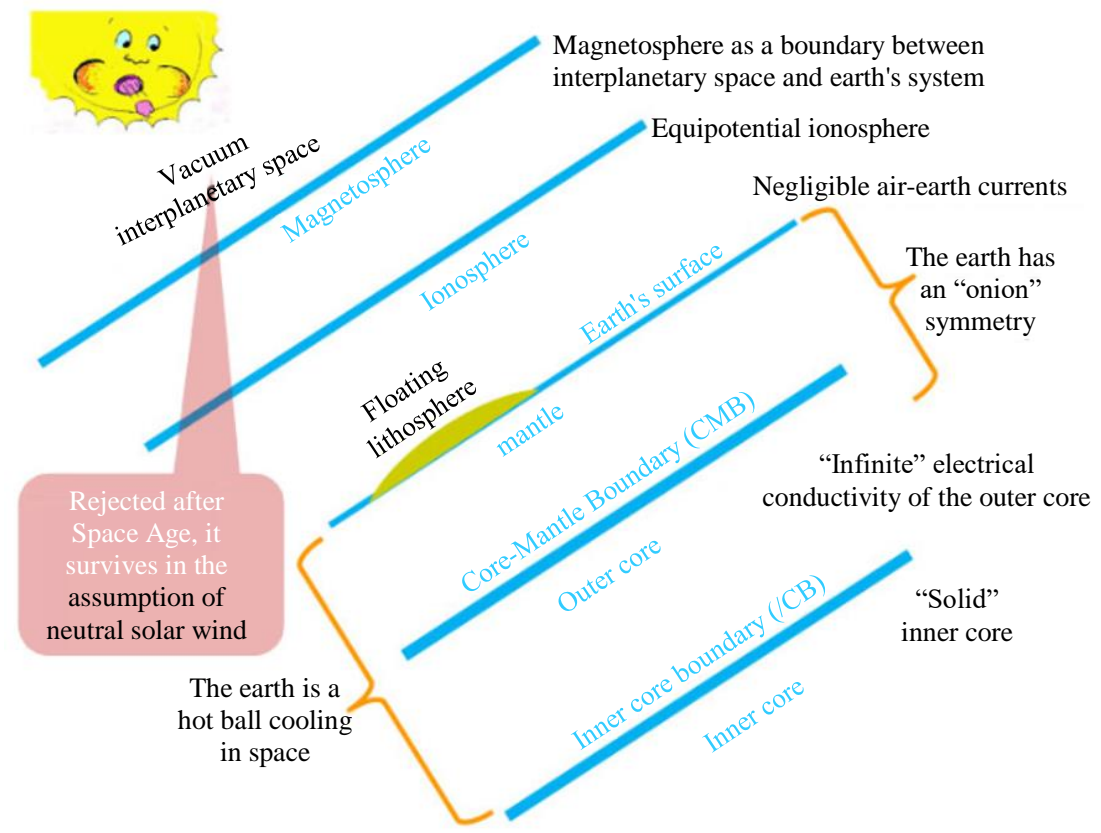

Fig. 27: Several unproven assumptions, that became real paradigms, are a present bias for understanding the Earth system. Several major "generally agreed" and unproven beliefs are here shown (in black). They represent effective "logical barriers" aimed to define and confine different disciplines. Figure after Gregori (2014). With kind permission of New Concepts in Global Tectonics

The solar wind is supposed to have a neutral charge, on the basis of a concept of the $1930 \mathrm{~s}$ when the existence of the solar wind was unknown. At present, several lines of evidence deny such an assumption ${ }^{52}$.

Quite a few ideal surfaces are supposed to be an almost impermeable separation for particle flow between contiguous space domains. In fact, the particles that are observed to cross through every such a surface are considered a lesser effect. This "artificial" and conventional belief leads to several incorrect paradigms, that are often basically aimed either to "simplify" models, or to separate "diplomatically" the competence of different specialists.

This criticism applies to the conventional definition (i) of the magnetopause, (ii) of the ionosphere, that is erroneously considered to an equipotential surface, just like in the case that it has an almost infinite electrical conductivity, (iii) of the Earth surface, where it is assumed that the effect of airearth currents can be neglected, consistently with a reasonable working assumption by Gauss, that, however, was never tested. Concerning the Earth's interior, a false belief is about a mean configuration that reminds about an onion. In fact, spherical layering matches the need for "simplicity" of mathematical algorithms. In contrast, nature knows no embarrassment by difficulties of analysis (Augustin-Jean Fresnel).

Another source of severe misunderstanding is the assumption that the $\mathrm{OC}$ has infinite electrical conductivity.

${ }^{52}$ Maybe, the best observational "proof" is the aforementioned peculiar orientation of the Pluto/Charon binary system and of their 4 minisatellites.
In addition, the generally and apparently unanimously agreed statement is reported that the IC is "solid". This is in contrast with every basic knowledge of solid-state physics. The state-of-matter at such high pressures and ionization is still to be clarified, being presumably related to the interaction between the magnetic moments of nuclei, when atoms lost-either entirely or almost entirelythe electron cloud.

Finally, the anachronism is becoming increasingly evident of patent-and copyright-regulation.

Concerning patents, two competing "doctrines" are to be recalled. Curie (and Sabin) claimed that a discovery is to be available free. In contrast, Nobel got huge patent rights and established the famous Nobel Prize.

The Covid-19 vaccines raised an unprecedented problem. If vaccination involves only one region or continent and not the neighboring population, the pandemics propagates also in vaccinated societies. Certainly, the patent rights must be protected of inventors and investors, but some limits have to be posed, upon considering several social and publichealth concerns.

Concerning copyright regulation, the present state-ofthe-art reminds about the confusion of units (for weight, length, surface, etc.) that existed in Europe before Napoleon, who imposed the metric system that later

Among the evidences is a straight explanation for the sunspot cycle as a periodic resetting of the electrostatic charge of the Sun. 
became the system generally used in science. ${ }^{53}$ At present, a jungle of sometimes conflicting rules makes quite arduous and annoying the job of a scientist who has to apply for permissions.

There is need to give the needed acknowledgement to authors and publishers. But, when dealing with study and research, all figures, data, tables, texts must be freely available. This is essential for the free circulation of ideas. Scientific institutions are other than commercial publishers. The present standards result into a true boycott for the progress of science and of understanding, hence-in the ultimate analysis-just for the capability of humankind to face the tremendous challenge of climate change, that-in the most extreme consequences-maybe could even represent a hazard for extinction of different species, including (perhaps) even mankind. The hazard and challenge cannot be neglected.

\section{Acknowledgement}

We thank Professor Qing-He Zhang for permission to use Fig. 24. It is impossible to mention all friends and colleagues who, at different times, contributed to the development and improvement of the interpretation and model that is here envisaged. We feel indebted particularly to (in alphabetical order) Franco Bonavia, Dong Wenjie, Gao Xiaoqing, Marvin Herndon, Martin Hovland, Alberto Incoronato, Louis J. Lanzerotti, Iginio Marson, Helmut Moritz, Nils-Axel Mörner, Cliff D. Ollier, Giuliano Panza, Michel Parrot, Kristian Schlegel, N. Christian Smoot, Karsten Storetvedt and the late Dong Choi, Arnaldo D'Amico, Naoshi Fukushima, Raymond Hide, Zbigniew Oziewicz, John M. Quinn and Wilfried Schröder.

\section{Author's Contributions}

Giovanni Pietro Gregori: Main development and management of the paper.

Bruce Allen Leybourne: Several substantial and intense discussions along the years during the exploitation of the full study. The text of the present paper was finally agreed in close cooperation between both authors.

\section{Ethics}

This article is original and contains unpublished material. Authors declare that are not ethical issues and no conflict of interest that may arise after the publication of this manuscript.

\footnotetext{
${ }^{53}$ The metric system was introduced in France in 1795 by the Convention, but had an eventful history for quite a few decades even in
}

\section{References}

Anonymous. (2008). Midocean ridges, Earthguide inline classroom, Earthguide at Scripps Institution of Oceanography.

Anonymous. (2018). Static gravity field-anomalies (1), Grace Tellus-NASA/JPL/Caltech Multimedia, retrieved 13 Aug 2018.

Budyko, M. I., Ronov, A. B., \& Yanshin, A. L. (1987). History of the Earth's atmosphere. 139 pp., SpringerVerlag.https://doi.org/10.1007/978-3-642-71674-4

Burn, W. (2015). Polygonal Crater formation by electrical discharges. New Concepts in Global Tectonics Journal, 3(2), 158-186.

Chester, F., (2014). The chestahedron-The wonder of seven-YouTube.

27.https://www.youtube.com/watch?v=dQMpEAsNH $\mathrm{mY}$

de Vries, M. V. W., Bingham, R. G., \& Hein, A. S. (2018). A new volcanic province: an inventory of subglacial volcanoes in West Antarctica. Geological Society, London, Special Publications, 461(1), 231-248. https://sp.lyellcollection.org/content/461/1/231.short.

Dong, W., \& Tang, M. (1997). A numerical modelling study on the processes of uplift and planation of the Tibetan Plateau. Sciencein China Series D: Earth Sciences, 40(3), 246-252. https://link.springer.com/content/pdf/10.1007/BF028 77532.pdf.

Frederikse, T., Landerer, F., Caron, L., Adhikari, S., Parkes, D., Humphrey, V. W.,... \& Wu, Y. H. (2020). The causes of sea-level rise since 1900. Nature, 584(7821),

393-397. https://www.nature.com/articles/s41586-020-2591-3

Gerringer, M. E., Linley, T. D., Jamieson, A. J., Goetze, E., \& Drazen, J. C. (2017). Pseudoliparis swirei sp. nov.: a newly-discovered hadal snailfish (Scorpaeniformes: Liparidae) from the Mariana Trench. Zootaxa, 4358(1), 161-177. https://www.mapress.com/j/zt/article/view/zootaxa.4 358.1.7

Gregori, G. P. (1992). Magnetospheric diagnostics by means of observations of polar auroras in Antarctica (electric field and plasma drift in the magnetosphere and in the polar ionosphere). 3rd Workshop Italian Research on Antarctic Atmosphere, Conference Proceeding, (pp. 361-374), SIF, Bologna.

Gregori, G. P. (1993). Geoelectromagnetism and geodynamics: 'corona discharge' from volcanic and geothermal areas. Phys. Earth Planet. Interiors, 77(1-2), 39-63.

https://www.sciencedirect.com/science/article/abs/pi i/0031920193900336.

France, before being adopted by many countries in the 19th century. We thank Pascal Richet (private communication). 
Gregori, G. P. (1997). Historical data and global change. In: Case Studies,Schröder, W. (ed.). Physics and geophysics with special historical case studies (A Festschrift in honour of K.-H. Wiederkehr). Mitteilungen des Arbeitskreises Geschichte der Geophysik der DDG, 16, Jahrgang (1997), (2/5) andNewsletter of IDCH-IAGA, (25), 1-409, Science Edition, Bremen Roennebeck and Potsdam. pp. 183-210.

Gregori, G. P. (1998). The magnetosphere of the Earth. A theory of magnetospheric substorms and of geomagnetic storms, pp. 68-106, in Schröder, W. (ed.) 1998. From Newton to Einstein-A Festschrift in honour of the 70th birthday of H.-J. Treder, Mitteilungen des Arbeitskreises Geschichte der Geophysik der DDG, 17, (3/4), 1-489, Science Edition, BremenRoennebeck and Potsdam.

Gregori, G. P. (1999). The external magnetic sources over the polar caps-Feasible modelling unrealistic expectations.Annali di Geofisica, 42(2), 171-189, https://www.earth-prints.org/handle/2122/1343

Gregori, G. P. (2001). Self-consciousness in Earth's sciences-Some personal reflections, pp. 123-133, in Schröder, W. (ed.) 2001. Wege zur Wissenschaft, Gelehrte erzählen aus ihrem Leben - Pathways to science, Scientists tell of their life and work, Beiträge zur Geschichte der Geophysik und Kosmischen Physik des Arbeitskreises Geschichte der Geophysik und Kosmischen Physik, (4), 283 pp., W. Schröder, AKGGKP, Bremen-Roennebeck.

Gregori, G. P. (2002). Galaxy-Sun-Earth relations. The origin of the magnetic field and of the endogenous energy of the Earth, with implications for volcanism, geodynamics and climate control and related items of concern for stars, planets, satellites and other planetary objects. A discussion in a prologue and two parts. Beitrage zur Geschichte der Geophysik und Kosmischen Physik, 3, pp; 471. https://www.dropbox.com/s/86hg9vsacuzz105/Gal axySunEarthRelationsGPGregori.pdf?dl=0.

Gregori, G. P. (2014). Climate and the atmospheric electrical circuit: the electromagnetic coupling between solar wind and Earth. New Concepts Global Tect. Journal, 2(1), 99-112.

Gregori, G. P. (2015). Anthropic global warming. New Concepts Global Technology Journa, 3, 544-560.

Gregori, G. P. (2020). Climate Change, Security, Sensors, Acoustics, 2(3), 474-504. Multidisciplinary Digital Publishing Institute.https://www.mdpi.com/2624$599 X / 2 / 3 / 26$

Gregori, G. P. and Dong, W. J., (1996). The correlation between the geomagnetic field reversals, the Hawaiian vulcanism and the motion of the Pacific plate. Annali di Geofisica, 39(1), 49-65.
Gregori, G. P., Bruce, A. L., \& Hissink, L. A. G. (2018). Natural "catastrophes": "forecast" and management deontological obligation and common sense, New Concepts Global Tect., 6(3), 327-346.

Gregori, G. P., Paparo, G., Poscolieri, M., Rafanelli, C., Ventrice, G., Garilli, G., Imperatori, L., Lo Castro, F., \& Zimatore, G. (2017). The seismic sequence in Central Italy (2016-2017) II-Problem solving and decision making on geodynamic interpretation, New Concepts Global Tect. J., 5(1), 92-110.

Heezen, B. C., \& Tharp, M. (1977). World ocean floor panorama. Full Color, Painted by H. Berann, Mercator Projection, Scale, 1(23,230,300).

Helman, M., Knott, S., Vergara, L., Richardsen, G., \& Karistrøm, E. (2001). Plate reconstructions of the North Atlantic region and the geological history of the Norwegian Sea. In Lunde, G. (ed.), 2001. Global wrench tectonics. New theory of Earth evolution. Proceedings of an International Workshop, Oslo, Norway, 9-11 May, 2001, website: http://www.earthevolution.org.

Janáčková, A., Urban, L., \& Krs, M. (1989). Geomagnetic field from the Earth's core: Its westward drift rate and some probable global-tectonics implications. Studia Geophysica et Geodaetica, 33(3), 246-253. https://link.springer.com/article/10.1007/BF01633528.

Judd, A. G., \& Hovland, M. (2007). Submarine fluid flow, the impact on geology, biology and the marine environment. 475 pp., Cambridge University Press.

Levenspiel, O. (2000). DEPARTMENTS-Learning from the Past-Earth's early atmosphere. Chemical Innovation, 30(5), 47-51.

Levenspiel, O., Fitzgerald, T. J., \& Pettit, D. (2000). Earth's atmosphere before the age of dinosaurs. Chemical Innovation, 30(12), 50-55.https://pascalfrancis.inist.fr/vibad/index.php?action=getRecordDe tail\&idt=824995.

Leybourne, B. A., \& Gregori, G. P. (2020). Introduction to plasma tectonics and electric geology: solar wind coupling to planetary circuits lightning tells the Stellar Transformer story, Journal of Systemics, Cybernetics and Informatics, 18(4), 7-13; http://www.iiisci.org/journal/sci/FullText.asp?var=\& id=ZA424OY20.

Leybourne, B. A., (2015). Earth as a stellar transformerClimate change revealed, New Conc. Global Tectonics J., 3(4), 365-366.

Leybourne, B. A., Davis, J. M., Gregori, G. P., Quinn, J. M., \& Smoot, N. C. (2017). Evolution of Earth as a stellar transformer, New Concepts Global Technology Journal, 5(1), 144-155.

MacKenzie, D. (1993). From the luminiferous ether to the Boeing 757: A history of the laser gyroscope. Technology and Culture, 34(3), 475-515. https://www.jstor.org/stable/3106702?seq=1 
Masalu, D. C. P. (2015). Global mid-ocean ridges mantle tomography profiles. Earth, 4(2), 80-88. https://doi.org/10.11648/j.earth.20150402.13

Mearns, E. (2015a). $\mathrm{CO}_{2}$-the view from space, energy matters, Posted on Oct 13, 2015. [CO2-the View from Space-Energy Matters.htm]

Mearns, E. (2015b). CO $\mathrm{CO}_{2}$-the view from space, energy matters, Posted on Jan 26, 2015. [CO2. the View from Space Energy Matters.htm].

Pappas, S. (2016). 500 'Champagne' methane seeps discovered off Pacific coast, Live Science issued Oct $20,2016$.

Quinn, J. M. (2014). Global remote sensing of Earth's magnetized lithosphere, 253 pp. Solar-Terrestrial Environmental Research Institute (STERI), Lakewood, CO.

Quinn, J. M., 2010. Global warming. Geophysical counterpoints to the enhanced greenhouse theory. 118 pp., Dorrance Publishing Co., Inc., Pittsburgh, USA.

Riguzzi, F., Crespi, M., Devoti, R., Doglioni, C., Pietrantonio, G., \& Pisani, A. R. (2012). Geodetic strain rate and earthquake size: new clues for seismic hazard studies. Phys. Earth Planet.Interiors, 206, 67-75. https://www.sciencedirect.com/science/article/abs/pi i/S0031920112001264

Ronov, A. B., (1982). The Earth's sedimentary shell. Quantitative patterns of its structure, compositions and evolution. The 20th V. I. Vernadskiy Lecture. Int. Geol. Rev., 24(11/12), 1313-1388. Translated from the Russian edition of 1980 (Izd-vo Nauka, Moscow, 80 pp.). Reprinted in 1983 by the American Geological Institute as a monograph, 80 pp. https://doi.org/10.1080/00206818209451075

Scott, D. E. (2015). Birkeland currents: a force-free fieldaligned model. Progress Phys., 11(2), 167-179.

Tang, M. C., \& Dong, W: J., (1997). Influences of seven Tibetan Plateau raising processes on climate and environment. Advance Atmosphere Science, 16(1), 23-29.

Tukey, J. W. (1977). Exploratory data analysis. 688 pp., Addison-Wesley Publ. Co., Reading. Massachusetts, etc.

Urban, L., \& Janáčková, A. (1990). A method of estimating the westward drift velocity and possible correlation with features of global tectonics. Phys.Earth Planet. Interiors, 59(4), 342-348. https://ui.adsabs.harvard.edu/abs/1990PEPI...59..342 U/abstract

Xing, Z. (2021). Prof. Zhang Qinghe's research group found the first known space hurricane poured electron rain, Shandong University News, issued Feb 28, 2021.
Zhang, Q. H., Zhang, Y. L., Wang, C., Oksavik, K., Lyons, L. R., Lockwood, M., \& Xia, L. D. (2021). A space hurricane over the Earth's polar ionosphere. Nature Communications, 12(1), 1-10. https://www.nature.com/articles/s41467-021-21459-y?

\section{Acronyms}

AAD-Australian Antarctic Discordance

AE-Auroral Electrojets (index)

ALB - Asthenosphere-Lithosphere Boundary

CMB - Core-Mantle Boundary

CME-Coronal Mass Ejection

EPR-East Pacific Rise

ESI-Electric Soldering Iron (mechanism)

FAC-Field-Aligned Currents

FI-Front Injection (mechanism for $\mathbf{E}$ generation inside the magnetosphere)

FR-Field Reversal (geomagnetic)

GPS-Global Positioning System

hbu-heartbeat unit

ICB-Inner Core Boundary

IC-Inner Core

IMF-Interplanetary Magnetic Field, synonymous of $\mathbf{B}_{\text {int }}$

IO-Ionosphere (mechanism for $\mathbf{E}$ generation inside the magnetosphere)

1.o.d.-length of the day

LIP-Large Igneous Province

MAR - Mid-Atlantic Ridge

MOR - Mid-Ocean Ridge

OC-outer core

OQR-orthogonal quasi-regular (solid object)

PIC-points of the inner circle

POC-points of the outer circle

SAA-South American Anomaly, synonymous with SAMA

SAMA-South Atlantic Magnetic Anomaly, synonymous with SAA

ss-spherical shell

SST-sea surface temperature

SV-secular variation (geomagnetic)

$\mathrm{SW}$-originated in the solar wind (mechanism for $\mathbf{E}$ generation inside the magnetosphere)

TD - tide-driven (dynamo)

TPA - trans-polar auroras

TP-tail plasma (mechanism for $\mathbf{E}$ generation inside the magnetosphere)

WHO-World Health Organization

WMT-warm mud tectonics 